Wiesław Dąbrowski ${ }^{1}$

Istituto Superiore delle Scienze Religiose L'Aquila

\title{
I frutti dello Spirito Santo - il commento di san Tommaso d'Aquino ai Gal 5,22ss
}

\section{Introduzione}

Nella Prima Secundae della Summa Theologiae( $\left.{ }^{2}\right)$, san Tommaso dedica ai frutti dello Spirito Santo, elencati in Gal 5,22, l'intera q. 70, dove spiega che i frutti dello Spirito Santo sono atti, quelli cioè che si compiono secondo la mozione dello Spirito Santo ${ }^{3}$; questi frutti vanno distinti dalle beatitudini, perché i frutti sono opere virtuose che procurano gaudio spirituale, mentre le beatitudini sono opere che procedono dai doni dello Spirito Santo ${ }^{4}$; i frutti dello Spirito Santo, elencati in Gal 5,22, scaturiscono in noi in quanto per la mozione dello Spirito Santo l'anima si dispone bene o in se stessa, o riguardo al prossimo o riguardo ai propri atti ${ }^{5}$ : essi ci fanno tendere al Cielo e perciò possono dirsi contrari alle opere della carne, che ci fanno tendere alle bassezze della terra ${ }^{6}$.

Questa sua Summa, però, benché la più diffusa e più studiata tra le sue opere, "non rappresenta l'insegnamento universitario di san Tommaso"? Egli infatti "non ha mai insegnato la Summa, ma la prima ora delle lezioni, chiamata ora Prima, quando la mente è ancora fresca, dedicava al commento dei testi ispirati”" ${ }^{\prime}$. Per

\footnotetext{
${ }^{1}$ Ksiądz Wiesław Dąbrowski, dr hab. teologii dogmatycznej, professore stabile ordinario teologii dogmatycznej w Wyższym Instytucie Nauk Religijnych w L'Aquila (ISSR-AQ), połączonym z Papieskim Uniwersytetem Lateraneńskim w Rzymie.

${ }^{2}$ Per quanto riguarda la questione che il vero titolo di questa Summa non è Summa Theologica, bensì Summa Theologiae, vedi A. Walz, De genuino titulo 'Summae theologiae', in 'Angelicum', 18 (1941), pp. 142-151.

${ }^{3}$ Vedi Summa Theologiae (più avanti come S.Th.), I-II, q. 70, a. 1.

${ }^{4}$ Vedi S.Th., I-II, q. 70, a. 2; vedi anche In Isaiam, c. 11.

${ }^{5}$ Vedi S.Th., I-II, q. 70, a. 3; vedi anche In III Sent., d. 34, q. 1, a. 5.

${ }^{6}$ Vedi S.Th., I-II, q. 70, a. 4.

${ }^{7}$ M.D. Chenu, Introduzione, in Tommaso d'Aquino, La conoscenza di Dio, Messaggero, Padova 1982, p. 5.

${ }^{8}$ Y.M.J. Congar, Zarys dziejów teologii, in AA.VV., Tajemnica Boga, Poznań-Warszawa-Lublin 1965, p. 190; vedi anche E.J. Gratsch, Manuale introduttivo alla Summa Teologica di Tommaso d'Aquino, Piemme, Casale Monferrato 1988, p. 24; I. Taurisano, La vita e l'epoca di san Tommaso d'Aquino, Studio Domenicano, Bologna 1991, p. 130; cfr. J.P. Torrell, Tommaso d'Aquino. L'uomo e il teologo, Piemme, Casale Monferrato 1994, pp. 169s.
} 
questo la teologia dell'Aquinate appare come "un'emanazione vitale e spiegazione della Sacra Pagina"'.

Nel Medioevo, infatti, il titolo ufficiale di professore di teologia era il magister in Sacra Pagina (solo alla fine del sec. XIII questo titolo fu cambiato in doctor in Sacra Theologia), ed il compito del magister era precisamente l'esposizione, cioè l'esegesi, della Sacra Scrittura ${ }^{10}$. Ed è per questo che "la teologia medievale ha padroneggiato i limiti, raggiungendo posizioni equilibrate e costruttive, perché nelle scuole medievali la Bibbia era intensamente letta e commentata e perché si viveva un concreto clima di fede"

San Tommaso, in veste di magister in Sacra Pagina, ha commentato il Corpus Paulinum due volte: la prima in Italia fra il 1259-1265, e poi a Napoli nel 1272-1273 o, come vogliono alcuni, a Parigi durante il suo secondo insegnamento negli anni 1269-1272 ${ }^{12}$. Così nacque il suo commento alle lettere di Paolo $\left({ }^{13}\right)$, cioè la sua Super Epistolas S. Pauli Lectura ${ }^{14}$.

È ovvio che "il Medio Evo in realtà non era in grado di praticare la critica storica con gli strumenti necessari, perché a questo tempo si studia il valore delle parole" 15 , tuttavia all'Aquinate non fu estranea non solo la storia dell'esegesi biblica, ma anche il metodo della critica letteraria e comparativa del testo sacro, i generi letterari e i sensi biblici ${ }^{16}$.

${ }^{9}$ M.D. Chenu, San Tommaso d'Aquino e la teologia, Gribaudi, Torino 1989, p. 26; vedi anche J. Duajat, in AA.VV., Aktualność św. Tomasza, Pax, Warszawa 1975, p. 12.

${ }^{10}$ Vedi per es. J.A. Weisheipl, Tommaso d'Aquino. Vita, pensiero, opere, Jaca Book, Milano 1994, pp. 50-136; O.H. Pesch, Tommaso d'Aquino. Limiti e grandezza della teologia medievale. Una introduzione, Queriniana, Brescia 1994, pp. 73s; J.P. Torrell, Tommaso d'Aquino. L'uomo e il teologo, Piemme, Casale Monferrato 1994, pp. 73-78.

${ }^{11}$ L. Serenthà, Teologia dogmatica, in Dizionario Teologico Interdisciplinare, Marietti, Torino 1977, vol. 1, pp. 262-278, qui p. 269; vedi anche J. Verger, L'esegesi dell'università, in AA.VV., Lo studio della Bibbia nel Medioevo latino, Paideia, Brescia 1989.

${ }_{12}$ Vedi per es. J.A. Weisheipl, Tommaso d'Aquino..., op. cit., pp. 250-254, 309, 380-381; J.P. Torrell, Tommaso d'Aquino..., op. cit., pp. 282-290, 379-380; O.H. Pesch, Tommaso d'Aquino..., op. cit., pp. $85,87 \mathrm{~s}$.

${ }^{13}$ San Tommaso era convinto che tutto il Corpus Paulinum fosse di Paolo; In Rom., Prologo, n. 11: "L'Apostolo scrisse quattordici lettere: nove di esse istruiscono la Chiesa dei Gentili; quattro i prelati ed i principi della Chiesa, cioè i reggenti; una il popolo di Israele, cioè quella che è /indirizzata/ agli Ebrei”; vedi anche In Hebr., Prologo, n. 4.

${ }^{14}$ In questo studio è usata l'edizione: S. Thomae Aquinatis, Super Epistolas S. Pauli Lectura (a cura di p. R. Cai OP, editio VIII revisa), 2 voll., Marietti, Torino-Roma 1953. Esiste l'edizione bilingue SAN Tommaso d'Aquino Commento al CORPUS PAULINUM, traduzione e introduzione di B. Mondin, voll. 1-6, Studio Domenicano, Bologna 2005-2008; tuttavia tutte le traduzioni delle citazioni di quest'opera di san Tommaso sono mie - W.D.

${ }^{15}$ H. Cazelles - J. P. Bouhot, Pentateuco, Paideia, Brescia 1968, p. 91.

${ }^{16}$ Vedi per es. In Hebr., Prologo, n. 5, dove san Tommaso si pronuncia a favore dell'autenticità paolina della Lettera agli Ebrei; vedi anche i luoghi indicati dall'Edizione Marietti 1953, vol. 2, Index rerum, voce: Critica textualis, pp. 563-564. Per quanto riguarda l'autore della Lettera agli Ebrei, vedi per es. S. Zedda, Lettera agli Ebrei. Introduzione, in P. Rossano (a cura di), Lettere di 
Come osserva B. Mondin, "l'esegesi biblica di s. Tommaso è estremamente precisa, analitica, rigorosa, ed arriva a vivisezionare il testo in maniera apparentemente impietosa, come se si trattasse di un'opera more geometrico demonstra$t a\left({ }^{17}\right)$ e, tuttavia, allo stesso tempo è un'esegesi contemplativa che sente e fa sentire il fascino della parola di Dio. Oltre il senso teologico, anche il senso morale e anagogico sono continuamente sottolineati così da fornire preziosi indirizzi alla vita spirituale del credente"18. E lo vedremo con molta facilità.

Prima però di passare al nostro tema, dobbiamo tener presente che anche se secondo l'Aquinate, "in ambedue le Scritture è contenuta quasi tutta la dottrina della teologia"19, poiché "la scienza divina in nessun luogo è trasmessa più competentemente che nella Sacra Scrittura" ${ }^{20}$ e perciò "soltanto la Scrittura canonica è regola della fede"21, tuttavia, commentando Eb 5,12: avete di nuovo bisogno che qualcuno v'insegni i primi elementi degli oracoli di Dio - rursum indigetis, ut vos doceamini, quae sint elementa exordii sermonum Dei( $\left.{ }^{22}\right)$, egli ci mette fortemente in guardia di fronte al pericolo del biblicismo $\left({ }^{23}\right)$ :

san Paolo, San Paolo, Cinisello Balsamo 1998, pp. 590-595, dove sono indicati, con i riferimenti patrologici, tutti i nomi accennati da san Tommaso nell'In Hebr., Prologo, n. 5.

${ }^{17}$ Per quanto riguarda la Super Epistolas S. Pauli Lectura, vedi l'Ed. Marietti 1953, Index synopticus-generalis, vol. 1, pp. 651-700; e vol. 2, pp. 507-553, dove ciò è presentato in modo grafico (nota - W.D.).

${ }^{18}$ B. Mondin, La cristologia di san Tommaso d'Aquino. Origine, dottrine principali, attualità, Urbaniana University Press, Vatican City 1997, p. 67.

${ }^{19}$ In Rom., Prol., n. 6.

${ }^{20}$ Super Boetium de Trinitate, q. 6, a. 2, ob. 1.

${ }^{21}$ Super Ioann., c. 21, lect. 6, n. 2656.

${ }^{22}$ In tali citazioni il testo latino proviene dalla Super Epistolas S. Pauli Lectura, mentre il testo italiano da La Bibbia di Gerusalemme, Dehoniane, Bologna 2009 (più avanti come BG; questa molto spesso si richiama a La Bible de Jérusalem, Paris 1998, più avanti come BJ); le citazioni bibliche nei testi di san Tommaso saranno tradotte quasi sempre dai testi biblici latini da lui riportati, il che permetterà di far vedere meglio come l'Aquinate intendeva quei testi e come essi funzionano nei suoi commenti. In alcuni casi i testi saranno confrontati anche con quelli della bibbia polacca, Biblia Tysiaclecia (La Bibbia del Millennio), Pallottinum, Poznań 2003 (più avanti come BT), ma - il che sottolineo fortemente - sempre come curiosum. Nel suo lavoro esegetico-teologico san Tommaso si serviva della cosiddetta Biblia Parisiensis, un'ottima edizione, per i tempi di allora, della Vulgata, curata dall'università di Parigi agli inizi del secolo XIII. Essa aveva l'ordine dei libri sacri come nelle nostre contemporanee moderne edizioni della Sacra Scrittura e la divisione in capitoli introdotta da Stefano Langton nel 1214, come la nostra. Migliorata poi e completata da Tommaso Gallo, l'ultimo grande esegeta della celebre scuola di San Vittore, che suddivise i capitoli in paragrafi, divenne l'edizione della "Bibbia dell'università di Parigi", che si è conservata fino ad oggi; vedi per es. C. Pera, Le fonti del pensiero di s. Tommaso d'Aquino nella Somma Teologica, Marietti, Torino 1979, p. 21; P. Richè - J. Chatillon - J. Verger, Lo Studio della Bibbia nel Medioevo latino, Brescia 1989, p. 95; B. Mondin, Dizionario enciclopedico del pensiero di san Tommaso d'Aquino, Ed. Studio Domenicano, Bologna 1991, voce: Bibbia, p. 96; G. D'onofrio (dir.), Storia della Teologia nel Medioevo, vol. 2: La grande fioritura, Piemme, Casale Monferrato 1996, pp. 200-203, 554-557.

${ }^{23}$ Oggi per es. F. Courth, Der Gott der dreifaltigen Liebe, ed. polacca: Bóg trójjedynej miło- 
Elementi chiamiamo quelle cose che nella grammatica vengono insegnate (traduntur) per primo, quando si tratta delle lettere; essi dunque sono le lettere stesse. Gli esordi dei sermoni di Dio, invece, ed i primi principi e gli elementi, sono gli articoli della fede e i precetti del Decalogo. Se uno dunque studiasse a lungo la teologia (diu studiuisset in theologia) e non conoscesse quelle cose, il tempo correrebbe contro di lui. Perciò l'Apostolo dice: avete bisogno che vi s'insegni quali sono gli elementi degli esordi dei sermoni di Dio, cioè i primi principi. 2 Tm 3,7: Dicono sempre e non arrivano mai alla scienza della verità. Is 65,20: Il ragazzo morirà a cento anni, il peccatore di cento anni sarà maledetto ${ }^{24}$.

Visto questo, non ci stupisce il fatto che, mentre le altre somme medievali erano "dirette agli intellettuali, ai grandi" 25 , san Tommaso scrisse la sua Somma di Teologia per "i principianti in teologia"26 (anche se lo studio di questa sua opera esige un'adeguata preparazione biblica, filosofica, teologica, patristica e giuridica ${ }^{27}$ ), e il Compendio di Teologia per coloro che sono troppo occupati nel lavoro e non hanno tempo per studiare la Sacra Scrittura ${ }^{28}$, e che tra le sue opere troviamo anche i commenti al Simbolo degli Apostoli, al Padre nostro, all' Ave Maria ed ai Due precetti della carità e ai Dieci comandamenti della Legge ${ }^{29}$.

A chi vorrebbe obiettare che questa è una teologia vecchia e oggi non interessa più nessuno, rispondo con le parole di Pietro Cardoletti, il quale sottolinea che "nel mondo culturale il vecchio, solo vecchio, totalmente e radicalmente vecchio, non può esistere, perché ogni lettura del vecchio è nuova, e il più accanito approfondimento del vecchio si trasforma sempre in una ultima novità, l'ultima

ści, Pallottinum, Poznań 1997, p. 15: "Il solo biblicismo non basta"; W. Kasper, Il Dio di Gesù Cristo, Queriniana, Brescia 2008, dopo aver presentato il problema di Dio oggi, dice in p. 22: "Come parlare quindi in modo comprensibile di Dio in questa situazione? Senz'altro non partendo immediatamente da una fede più o meno ovvia in Dio, ma nemmeno procedendo in termini meramente positivistici dal Dio della rivelazione biblica".

${ }^{24}$ In Hebr., c. 5, lect. 2, n. 266. Studiuisset in theologia: I. Biffi, Teologia, storia e contemplazione in Tommaso d'Aquino, Jaca Book, Milano 1995, p. 145, annovera questo importante testo (studere in theologia) solo tra le "espressioni linguistiche" riguardanti la theologia. $2 \mathrm{Tm}$ 3,7: Vlg: Semper discentes, et numquam ad scientiam veritatis pervenientes; BG: Stanno sempre lì ad imparare, senza riuscire mai a giungere alla conoscenza della verità. - Is 65,20: Vlg: Puer centum annorum morietur, peccator centum annorum maledictus erit; BG: Poiché il più giovane morirà a cento anni e chi non raggiunge i cento anni sarà considerato maledetto; BT: ... e il non raggiungere i cento anni sarà segno della maledizione.

${ }^{25}$ I. Taurisano, La vita e l'epoca di san Tommaso d'Aquino, op. cit., p. 130.

${ }^{26}$ Vedi S.Th., I, Prologo.

${ }^{27}$ Vedi E.J. Gratsch, Manuale introduttivo alla Summa Teologica di Tommaso d'Aquino, op. cit., p. 33.

${ }^{28}$ Vedi Comp. Theol., Prologo, n. 1; traduzione italiana: S. Tommaso d'Aquino, Compendio di Teologia, introduzione, traduzione e note a cura di P. Agostino Selva OP, Studio Domenicano, Bologna 1995.

${ }^{29}$ Traduzione italiana: S. Tommaso d'Aquino, Opuscoli spirituali. Commenti al Credo, al Padre nostro, all'Ave Maria e ai Dieci Comandamenti, traduzione e note a cura di P. Lippini, Studio Domenicano, Bologna 1999. 
interpretazione. Il vecchio è completamente vecchio quando non è più letto, quando è assenza dal nostro mondo" 30 .

Adesso passiamo al nostro tema.

La lista dei frutti dello Spirito in Gal 5,22-23 è posta in contrasto rispetto ad una delle cosiddette "liste dei vizi" ${ }^{1}$ o "cataloghi dei delitti" 32 in Gal 5,19-21, che Paolo "adatta al suo conteso" 33 , perciò vale la pena di vedere come l'Aquinate commenta questo testo in cui è evidente che "già nel periodo neotestamentario è maturata la coscienza che esistono i peccati, i quali escludono dal regno di Dio (cfr. l'elenco dei vizi in Rm 1,29-32; 1 Cor 6,9s; Gal 5,19-21) e, a differenza di altri peccati (più leggeri), conducono al giudizio e alla morte eterna (1 Gv 5,16)"34.

Gli elenchi delle virtù e dei vizi, che troviamo nelle lettere di Paolo - afferma C.G. Kruse - "intendono spronare i lettori ad abbandonare i vizi elencati e a praticare le virtù" 35 . Infatti, in Gal 5,19-23 l'Apostolo "ricorda ai suoi lettori che la libertà dalla Legge non significa assecondare i desideri della carne (elencati in Gal 5,19-21); essa deve invece condurre alla manifestazione del frutto dello Spirito (specificato in dettaglio in Gal 5,22-23)" ${ }^{\prime 36}$.

${ }^{30}$ P. Cardoletti, Presentazione, in B. Lonergan, Conoscenza e interiorità. Il Verbum nel pensiero di s. Tommaso, Dehoniane, Bologna 1984, p. 10; cfr. G.L. Brena, Interpretazione antropologica di san Tommaso, in AA.VV., Tommaso d'Aquino nel suo settimo centenario, vol. 7: L'uomo, Ed. Domenicane Italiane, Napoli 1978, pp. 83-100, qui p. 89: "Rivolgere le nostre domande a un pensatore del passato è gettare un ponte (...), e superiamo la precomprensione del passato verso una esplicitazione accurata del nostro orizzonte culturale, nelle somiglianze e differenze dal contesto antico. Così viene circostanziato e motivato, mediante la distanza storica, il nuovo senso e valore attuale che assume per noi il pensiero degli antichi"; S. Swieżawski, Święty Tomasz na nowo odczytany, Kraków 1983, p. 23: "Proprio ciò che perennemente rimane giovane, rimane anche perennemente attuale".

${ }^{31}$ Così la BG, ed. cit., p. 2681, chiama Rm 1, 29-32 e parall., tra cui Gal 5,19ss.

${ }^{32}$ Così la BT, ed. cit., p. 1300, chiama Rm 1, 29-32 e parall., tra cui Gal 5,19ss, p. 1347. Tutte le "liste dei vizi" con le interpretazioni di san Tommaso, vedi: W. Dąbrowski, La dottrina sul peccato originale nei commenti di san Tommaso d'Aquino alle lettere di san Paolo Apostolo, in 'Angelicum' 83 (2006), pp. 557-629, qui pp. 568-589.

${ }^{33}$ C.G. Kruse, Virtù e vizi, in G.F. Hawthorne - R.P. Martin - D.G. Reid (a cura di), Dizionario di Paolo e delle sue lettere, San Paolo, Cinisello Balsamo 2000, pp. 1605-1607, qui p. 1607.

${ }^{34}$ G.L. Müller, Dogmatica cattolica. Per uno studio e la prassi della teologia, San Paolo, Cinisello Balsamo 1999, p. 874.

${ }^{35}$ C.G. Kruse, Virtù e vizi, op. cit., p. 1605; vedi anche H. Währisch - A. Tessarolo, Virtù, in L. Coenen - E. Beyreuther - H. Bietenhard (a cura di), Dizionario dei concetti biblici del Nuovo Testamento, Dehoniane, Bologna 1991, pp. 1989-1993; B. Gillièron, Lessico dei termini biblici, Elledici, Leumann 2000, voce: Virtù, pp. 302-303; X. Leon-Dufour, Dizionario del Nuovo Testamento, Queriniana, Brescia 1978, voce: Virtù, pp. 553-554; voce: Vizi, pp. 556-557; H. Obermayer - K. Speidel - K. Vogt - G. Zieler, Piccolo dizionario biblico, Paoline, Cinisello Balsamo 1991, voce: Virtù, p. 339; M.-F. Lacan, Virtù e vizi, in X. Leon-Dufour (dir.), Dizionario di teologia biblica, Marietti, Genova 2001, coll. 1381-1384.

${ }^{36}$ C.G. Kruse, Virtù e vizi, op. cit., p. 1605. 


\section{I vizi - Gal 5,19-21}

Del resto - leggiamo in Gal 5,19-21 - sono ben note le opere della carne: fornicazione, impurità, dissolutezza, idolatria, stregonerie, inimicizie, discordia, gelosia, dissensi, divisioni, fazioni, invidie, ubriachezze, orge e cose del genere. Riguardo a queste cose vi preavviso, come già ho detto: chi le compie non erediterà il regno di Dio - Manifesta sunt autem opera carnis, quae sunt fornicatio, immunditia, impudicitia, luxuria, idolorum servitus, veneficia, inimicitiae, contemptiones, aemulationes, irae, rixae, dissensiones, sectae, invidiae, homicidia, ebrietates, comessationes, et his similia, quae praedico vobis, sicut praedixi, quoniam qui talia agunt, regnum Dei non consequentur $\left({ }^{37}\right)$.

U. Vanni, seguendo S. Zedda ${ }^{38}$, raggruppa questa "lunga enumerazione" in quattro categorie: "Peccati di lussuria: fornicazione: cioè matrimoni irregolari e relazioni sessuali illecite; impurità: si parte dal concetto di una sordidezza materiale trasportandolo nel campo morale con significato generico; dissolutezza: sfrenatezza nel campo sessuale. Peccati contro la religione: idolatria; magia: lett. uso di droghe, da cui il senso di incantesimo, stregoneria. Peccati contro la carità: inimicizie: ostilità che partono dall'interno e si manifestano al di fuori nei modi più disparati; lite: contesa litigiosa; gelosia: intesa talvolta anche in senso buono, qui, dal contesto, ha il significato di « un sentimento ostile eccitato da un bene posseduto da altri »(Burton, 30779); ire: lett. respiro, animo: in senso traslato anche coraggio e, in senso peggiorativo come qui, alterigia; ambizioni: di etimologia incerta, anche se il termine greco è collegato sempre con guadagno; potremmo dire: ambizione egoistica, o semplicemente egoismo, ricerca di sé; divisioni: dissensioni, parti in contrasto; più tardi il termine sarà usato nel senso del nostro 'eresia'; invidie: cattiva volontà, malizia ei riguardi degli altri; il plurale indica una molteplicità di atti. Peccati contro la temperanza: ubriachezze, orge conviviali. Rispetto a tutte queste azioni Paolo ricorda con la massima chiarezza quanto ha già detto prima a voce: esse escludono dall'eredità escatologica del regno di Dio. Con ciò Paolo sottolinea la gravità delle singole azioni e del seguire la carne in generale" 40 .

Secondo san Tommaso, l'Apostolo indica qui le opere della carne, proibite anche dalla Legge, che si oppongono allo Spirito Santo.

Prima però di esaminare queste opere della carne, l'Aquinate risolve tre dubbi. Il primo dubbio concerne il fatto che qui l'Apostolo enumera alcuni peccati

${ }^{37}$ BG, nota a Gal 5,20: "gelosia: la volg. e altri aggiungono: omicidi (cfr. Rm 1,29)"; vedi anche U. Vanni (versione - introduzione - note), Lettere ai Galati e ai Romani, in Nuovissima versione della Bibbia, vol. 40, Paoline, Roma 1979, p. 62.

${ }^{38}$ Vedi S. Zedda, Prima Lettura di S. Paolo, Torino 1964, p. 87.

${ }^{39}$ E.W. De Burton, A Critical and Exegetical Commentary on the Epistle to the Galatians, Edinburgh 1921, p. 307.

${ }^{40}$ U. Vanni, Lettera ai Galati, in P. Rossano (a cura di), Lettere di san Paolo, op. cit., p. 257. 
che non riguardano la carne, ma tuttavia le chiama opere della carne, cioè l'idolatria, le sette, le rivalità ed altre di tale tipo. Ed ecco la sua risposta, attuale anche oggi, basata sull'autorità di un grande Padre della Chiesa:

Secondo Agostino - La Città di Dio, lib. 14, c. 2 - vive secondo la carne chiunque vive secondo se stesso. Perciò per carne si intende qui tutto l'uomo. Qualunque cosa, dunque, provenga dal disordinato amore proprio si chiama opera della carne. Oppure bisogna dire che qualche peccato si può dire carnale duplicemente, cioè quanto alla consumazione: e così si dicono carnali soltanto quei /peccati/ che si consumano nel godimento della carne, cioè lussuria e gola; e quanto alla radice: e così tutti i peccati si dicono carnali, in quanto dalla corruzione della carne viene appesantita l'anima, come è detto in Sap 9,15, e da ciò indebolito l'intelletto può essere più facilmente ingannato e impedito nella sua perfetta operazione. Perciò anche da ciò seguono i vizi, cioè le eresie, le sette ed altri di tale tipo. $\mathrm{E}$ in questo modo si dice che il fomento è il principio di ogni peccato ${ }^{41}$.

Il secondo dubbio concerne il fatto che nessuno è escluso dal regno di Dio se non per il peccato mortale, perciò dalle parole: coloro che fanno tali cose non conseguiranno il regno di Dio, risulterebbe che tutti i peccati soprannominati siano peccati mortali, mentre si vede che molti di essi non sono peccati mortali, come la contesa, la rivalità ed altri di tale tipo. E l'Angelico risponde:

Tutti quei /peccati/ che sono stati enumerati, sono in qualche modo mortali; alcuni però secondo il loro genere, come l'omicidio, la fornicazione, l'idolatria e di tale tipo; alcuni invece secondo la loro consumazione, come l'ira, la cui consumazione sta nel danno del prossimo. Perciò, se si aggiunge il consenso del danno stesso, è peccato mortale. E similmente il banchetto (comestio) è ordinato al godimento del cibo; se però uno pone il suo fine nel godimento di tale tipo, pecca mortalmente: e perciò l'Apostolo non dice "banchetti", ma gozzoviglie; e similmente si intende di altri simili $/$ peccati ${ }^{/ 2}$.

Il terzo dubbio concerne l'ordine e l'enumerazione di quei vizi-peccati. Ed ecco la risposta:

Quando l'Apostolo, in diversi luoghi, enumera diversi vizi in diversi modi, non intende enumerare tutti i vizi ordinatamente e secondo un sistema (secundum artem),

${ }^{41}$ In Gal., c. 5, lect. 5, n. 320; vedi anche S.Th., I-II, q. 72, a. 2 (Utrum convenienter distinguantur peccata spiritualia a carnalia; resp. - sì; nell'ob. 1 c'è Gal 5,19ss; cfr. ad 1); q. 73, a. 5; II-II, a. 118, a. 6; In 1 Cor., c. 6, lect. 3, n. 307; In 2 Cor., c. 7, lect. 1, n. 246. S. Agostino, De Civ. Dei, lib. 14, c. 2 (PL 41, 404; c. 3: PL 41, 406 - compl. W.D.); vedi anche Glossa ordin., super Gal. 5,19 (VI 87 E); Pietro Lombardo, Glossa super Gal. 5,19 (PL 192, 159) (nota - W.D.). Sap 9,15: BG: Perché un corpo corruttibile appesantisce l'anima e la tenda d'argilla opprime una mente piena di preoccupazioni; BT: Perché un corpo mortale schiaccia l'anima e la tenda terrena appesantisce la ragione piena di pensieri.

${ }^{42}$ In Gal., c. 5, lect. 5, n. 321; vedi anche S.Th., I-II, q. 73, a. 5 (Utrum peccata carnalia sint minoris culpae quam spiritualia; resp. - sì); II-II, q. 154, a. 3; In IV Sent., d. 33, q. 1, a. 3, qc. 2, ad 3; De Veritate, q. 25, a. 6, ad 2; In Isaiam, c. 1. 
ma soltanto quelli di cui abbondano ed in cui eccedono coloro ai quali scrive. E perciò non bisogna cercare in essi la sufficienza, ma la causa della diversità ${ }^{43}$.

I vizi-peccati elencati in Gal 5,19-21, il nostro teologo-esegeta li divide in due gruppi: quelli che riguardano le cose non necessarie alla vita, e quelli che riguardano le cose necessarie alla vita. Questo primo gruppo è diviso ancora in tre: i peccati contro se stessi, i peccati contro Dio e i peccati contro il prossimo, evidenziando così un fatto molto importante, cioè che i vizi-peccati hanno non solo una dimensione personale, ma anche quella teologica e quella sociale-ecclesiale.

I vizi-peccati contro se stessi - continua san Tommaso - l'Apostolo li pone al primo posto, perché evidentemente procedono dalla carne, e sono quattro:

Due di essi si riferiscono all'atto carnale della lussuria, cioè la fornicazione, che avviene quando un solitario accede ad una solitaria, o quanto all'uso naturale della lussuria. L'altro è l'immondizia quanto all'uso contro natura. Ef 5,5: Ogni fornicatore o immondo, ecc $\left({ }^{44}\right)$. 2 Cor 12,21: E non fecero penitenza per l'immondizia

${ }^{43}$ In Gal., c. 5, lect. 5, n. 322.

${ }^{44}$ In Eph., c. 5, lect. 2, nn. 277-280 (Ef 5,5: Perché, sappiatelo bene, nessun fornicatore, o impuro, o avaro - cioè nessun idolatra - ha in eredità il regno di Cristo e di Dio - Hoc enim scitote, intelligentes, quod omnis fornicator, aut immundus, aut avarus, quod est idolorum servitus, non habet haereditatem in regno Christi et Dei): L'Apostolo dunque dice: Sappiatelo, intelligenti, cioè attualmente, non solo abitualmente, l'abbiate per cosa certa. $1 \mathrm{Gv} 5,13$ : Vi ho scritte queste cose, perché sappiate, ecc. E che cosa? Che ogni fornicatore, o immondo, o avaro, il che è servizio degli idoli, non ha l'eredità nel regno di Cristo e di Dio. Nota che qui l'Apostolo chiama l'avarizia idolatria, perché l'idolatria c'è quando l'onore debito a Dio solo viene dato alla creatura. Adesso invece si deve a Dio un duplice onore, che cioè in Lui costituiamo il nostro fine e che finalmente poniamo in Lui la nostra fiducia; chi dunque pone ciò nelle creature è reo di idolatria. Ciò invece fa l'avaro che pone nella cosa creata il suo fine ed anche tutta la sua fiducia. Os 8,4: Si fecero idoli il loro argento e il loro oro. E ciò perché, come è detto in Pr 11,28: Chi confida nelle sue ricchezze cadrà. Ma, visto che in altri peccati l'uomo pone il suo fine nella creatura, perché anche in quelli il peccatore non è chiamato idolatra? Rispondo. Idolatrare è dare esteriormente il culto indebitamente a qualche cosa. Ora invece in altri peccati il fine è posto nelle cose interiori quasi nella propria esaltazione. Ma chi pone il fine nelle ricchezze, pone il fine in esse come nella cosa esteriore, come /lo fa/ l'idolatra. Ma gli avari, che l'onore dovuto a Dio offrono (exhibentes) alla creatura, sono idolatri realmente e di per sé? Dico che no, perché negli atti, cioè nelle opere morali, vengono giudicati dal fine. Dunque, è di per sé idolatra chi di per sé ha l'intenzione di offrire (exhibere) il culto alla creatura. Ciò l'avaro non ha l'intenzione di farlo di per sé, ma lo fa per accidens, in quanto ama eccessivamente (superflue) e disordinatamente. E che cosa di un tale /dice l'Apostolo/? Non avrà l'eredità, davvero, perché figli ed eredi, come è detto in $\mathrm{Rm} 8,17$. Adesso invece i tali, che sono così carnali, non sono figli; dunque non hanno l'eredità, perché, come è detto in 1 Cor 15,50: La carne e il sangue non possiederanno il regno di Dio, cioè Dio, il quale dice in Ez 44,28: Io sono la loro eredità. Ma si potrebbe domandare: Se questa eredità è Dio stesso, il quale è indivisibile e impartibile, perché l'Apostolo dice: nel regno di Cristo e di Dio, dividendo, come se questa eredità fosse divisibile? Rispondo. La nostra eredità consiste nella fruitio Dei, adesso invece Dio gode se stesso in un altro modo (aliter se 
e la fornicazione e la impudicizia, ecc $\left({ }^{45}\right)$. Gli altri due sono ordinati agli atti stessi. L'uno, cioè esteriore, come i toccamenti, gli sguardi, i baci e di tale tipo; e quanto a ciò dice: impudicizia, Ef 4,19: I quali, disperati, hanno consegnato se stessi all'impudicizia, ecc $\left.{ }^{46}\right)$. L'altro - interiore, cioè nei pensieri immondi; e quanto a ciò

fruitur) e noi lo /godiamo in un altro modo/; siccome Dio gode se stesso perfettamente, perché perfettamente conosce se stesso ed ama quanto è conoscibile ed amabile. Noi invece non così, perché giustamente Lo conosceremo perfettamente in patria e, per conseguenza, l'ameremo, perché chi raggiunge qualche cosa semplice, la conosce tutta, e se non totalmente, come la luce del sole - se fosse puntuale, l'occhio umano l'apprenderebbe tutta - non /la comprendiamo/ totalmente, mentre l'occhio dell'aquila la comprenderebbe totalmente. Così, anche se in patria conosceremo Dio perfettamente e perfettamente l'ameremo, ma non lo comprenderemo totalmente, perciò si vede che là c'è qualche imperfezione e particolarità. E perciò l'Apostolo dice congiuntamente: di Cristo e di Dio, ponendo quasi parte con parte, cioè perché per mezzo di Cristo e non per mezzo di un altro si ha l'eredità. Os 8,4: BG e BT: Con il loro argento e il loro oro si sono fatti idoli.

${ }^{45}$ In 2 Cor., c. 12, lect. 6, n. 514 (2 Cor 12,21: E non si sono convertiti dalle impurità, dalle immoralità e dalle dissolutezze che hanno commesso - Et non egerunt poenitentiam super immunditia, et fornicatione, et impudicitia quam gesserunt): Qui l'Apostolo manifesta la loro malizia quanto ai mali passati, dei quali non si sono pentiti, (...) e non fecero penitenza, pienamente dopo la prima lettera. E giustamente piango, perché come la gloria del padre è la gloria dei figli, così la confusione del padre è la confusione dei figli. Così Samuele piangeva Saul in 1 Sam 16,1: Fino a quando piangerai su Saul, ecc. E ciò perché non si sono pentiti né fecero penitenza per i suddetti peccati carnali, di cui alcuni sono contro natura. E perciò dice: per l'immondizia cioè per la lussuria contro natura. Alcuni /di quei peccati/ sono commessi con le donne corrotte, cioè con le vedove o le coniugate, e perciò dice e per la fornicazione. Alcuni /di quei peccati/ diventano corruzione delle vergini, e perciò dice: e per l'impudicizia che avevano commesso. Gal 5,19: Le opere della carne sono manifeste: la fornicazione, l'impurità, l'impudicizia, ecc.

${ }^{46}$ In Eph., c. 4, lect. 6, n. 235-236 (Ef 4,19: Così, diventati insensibili, si sono abbandonati alla dissolutezza - Qui desperantes semetipsos tradiderunt impudicitiae; BG nota: "Così, diventati insensibili: la volg. ha: avendo perduto ogni speranza"; BT: condottisi all'insensibilità [della coscienza]): Seguono le parole: I quali, disperati ecc., dove l'Apostolo fa vedere /agli Efesini/ quali erano nel comportamento esteriore, cioè senza speranza, e ciò perché erano estranei alla vita /di Dio/ (alienati a vita /Dei/: vedi Ef 4,18; In Eph., n. 233 - nota W.D.). Gb 7,16: Sono disperato, perché già non vivrò oltre, ecc. Ger 18,12: Siamo disperati, andiamo infatti dietro le nostre cogitazioni, e facciamo del male ciascuno secondo la malvagità del suo cuore. E questo è ciò che segue: Hanno consegnato se stessi all'impudicizia, ecc. (...) perché, in primo luogo, hanno peccato non per passione, ma piuttosto per scelta, perciò l'Apostolo dice: hanno consegnato se stessi all'impudicizia; come se dicesse: Non hanno peccato per passione o per debolezza, ma hanno consegnato se stessi ecc. Gdt 7,15: Spontaneamente tutti ci consegniamo al popolo di Oloferne, ecc. 2 Cor 12,21: E non fecero penitenza per l'impudicizia che hanno commesso, ecc. Gb 7,16: Vlg: Desperavi, nequaquam ultra iam vivam; BG: Mi sto consumando, non vivrò più a lungo; BT: Perirò. Non vivrò in eterno. - Ger 18,12: Vlg: Desperavimus, post cogitationes enim nostras ibimus, et unusquisque post pravitatem cordis sui malefaciemus; BG: $\grave{E}$ inutile, noi vogliamo seguire $i$ nostri progetti, ognuno di noi caparbiamente secondo il suo cuore malvagio; BT: cuore perverso. - Gdt 7,26/15/: BG e BT: Ormai chiamateli e consegnate l'intera città al popolo di Oloferne. 
dice: lussuria, $1 \mathrm{Tm} 5,11\left({ }^{47}\right)$ : Quando infatti saranno prese dalla lussuria in Cristo, vogliono sposarsi, $\mathrm{ecc}^{48}$.

Contro Dio - l'Aquinate continua la sua lezione, e possiamo confrontarla con quella di U. Vanni e S. Zedda (Peccati contro la religione ${ }^{49}$ ) - l'Apostolo pone due peccati:

Uno di essi consiste nell'impedire, da parte degli avversari di Dio, il culto divino; e quanto a ciò dice: servizio degli idoli, 1 Cor 10,7: Non diventate idolàtri, ecc. Sap 14,27: Il culto di idoli nefandi è causa, inizio e fine d'ogni male. L'altro consiste nell'entrare nel patto con il demonio; e quanto a ciò dice: venefici-stregonerie, che avvengono per mezzo delle arti magiche e si chiamano venefici-stregonerie dal veleno-incantesimo (dicuntur veneficia a veneno), perché sono a danno degli uomini. 1 Cor 10,20: Non voglio che diventiate soci dei demoni. Ap 22,15: Fuori i cani ed $i$ venefici, ecc ${ }^{50}$.

Contro il prossimo - leggiamo nell'esposizione dell'Angelico - l'Apostolo pone nove peccati, il primo dei quali è l'inimicizia, l'ultimo è l'omicidio, perché a questo si arriva da quello (notiamo qui ben descritto il processo psicologico-spirituale di questi vizi-peccati - nota W.D.):

Il primo dunque è l'inimicizia nel cuore, che è l'odio verso il prossimo. Mt 10,36: Nemici dell'uomo i suoi domestici. E perciò dice: inimicizie. Da questa, poi, prende l'origine il dissenso nelle parole. E perciò dice: contese, che è "l'attacco alla verità con confidenza nel clamore". Pr 20,3: $\grave{E}$ un onore per l'uomo se si separa dalle contese. Il secondo /vizio/ è la rivalità che consiste nel fatto che per sostenere uno si gareggia con un altro. Perciò l'Apostolo dice: rivalità, che provengono dalle contese. Il terzo è quando uno viene impedito da un altro tendente verso la stessa cosa e da ciò si adira contro di lui, e perciò dice: ire, Gc 1,20: Perché l'ira dell'uomo, ecc. Ef

${ }^{47}$ In 1 Tim., c. 5, lect. 2, nn. 202-203 (1 Tm 5,11: Quando vogliono sposarsi di nuovo, abbandonano Cristo - Cum enim luxuriatae fuerint in Christo, nubere volunt): Qui l'Apostolo indica la duplice ragione dal duplice pericolo che minaccia /le vedove più giovani/. (...) Se infatti le /vedo$\mathrm{ve} /$ più giovani (adolescentes) vengono assunte al sostentamento della Chiesa, ce ne sono due conseguenze, cioè che avranno ciò che è sufficiente e che non si metteranno al lavoro (non cogantur manibus operari). Da entrambe le cose, invece, minaccia il pericolo. Dalla prima, il pericolo alla castità, perciò dice: quando infatti saranno prese dalla lussuria. Per lussuria talvolta s'intende l'eccesso degli atti sessuali (sumitur pro superfluitate actus venerei), e così è uno dei stette vizi capitali; talvolta invece - ogni superfluità delle cose corporali, e così s'intende qui; come se l'Apostolo dicesse: Quando avranno la sovrabbondanza in Cristo, cioè grazie all'aiuto di Cristo, allora vogliono sposarsi. Es 32,6: Il popolo sedette per mangiare e bere, e poi si alzò per divertirsi.

${ }^{48}$ In Gal., c. 5, lect. 6, n. 323; vedi anche S.Th., II-II, q. 150, a. 1, ad 1; a. 3; q. 154, a. 2; In III Sent., d. 34, q. 1, a. 5; De Malo, q. 15, a. 2, sed contra.

${ }^{49}$ Vedi U. Vanni, Lettera ai Galati, in P. Rossano (a cura di), Lettere di san Paolo, op. cit., p. 257.

${ }^{50}$ In Gal., c. 5, lect. 6, n. 324. Ap 22,15: BG: Fuori i cani, i maghi, gli immorali, gli omicidi, gli idolàtri e chiunque ama e pratica la menzogna; BT: Fuori i cani, gli indovini, i libertini, gli omicidi, gli idolàtri e chiunque ama la menzogna e vive di essa. 
4,26: Non tramonti il sole sopra la vostra iracondia $\left.{ }^{51}\right)$. Il quarto, quando dall'ira dell'animo si perviene alle percosse; e quanto a ciò dice: risse. Pr 10,12: L'odio suscita risse. Il quinto: dissensi, e se riguardano le faccende umane (si in rebus humanis sint), si chiamano dissensi, quando cioè ci sono parzialità nella Chiesa. Rm 16,17: Osservate coloro che fanno dissensi ed ostacoli davanti alla dottrina che voi avete imparato, e tenetevi lontani da loro $\left({ }^{52}\right)$. Se nelle cose divine, allora si chiamano sette,

${ }^{51}$ In Eph., c. 4, lect. 8, n. 251 (Ef 4,26: Non tramonti il sole sopra la vostra ira - Sol non occidat super iracundiam vestram): Qui l'Apostolo espone ciò che ha detto (vedi Ef 4,26 all'inizio; In Eph., n. 250 - nota W.D.), e, secondo le tre precedenti esposizioni, lo si può esporre triplicemente, perché se /si tratta/ dell'ira cattiva, allora così: Non tramonti il sole ecc., cioè: Non persistete nell'ira concepita, ma deponetela prima del tramonto del sole, perché sebbene sia permesso il moto, a motivo della fragilità, non è permesso il ritardo. Se /si tratta dell'ira/ buona, e ciò contro i propri peccati, allora così: Non tramonti il sole, cioè il Cristo, Ml 4,2: Per voi, che temete il mio nome, sorgerà il sole di giustizia, ecc., sopra la vostra iracondia, cioè sopra i vostri peccati, per i quali bisogna che adesso vi adiriate e puniate voi stessi. Se /si tratta dell'ira/ contro i peccati degli altri, si interpreta così: Non tramonti il sole, cioè della ragione. Qo 12,1s: Ricordati del tuo creatore nei giorni della tua giovinezza, prima che venga il tempo dell'afflizione e si avvicinino gli anni dei quali dovrai dire: "Non mi piacciono"; prima che si ottenebri il sole, ecc. Non tramonti il sole sopra la vostra iracondia, cioè non si ottenebri il dettame della ragione. Gb 5,2: L'iracondia uccide l'uomo stolto. Qo 12,1s: BG: Ricordati del tuo creatore... i giorni tristi e giungano gli anni...: "Non ci provo alcun gusto"; prima che si oscurino il sole, la luce, la luna e le stelle; BT: ... i giorni dell'afflizione.... "Non mi compiaccio in essi", ecc. - Gb 5,2: BG: La collera uccide lo stolto; BT: L'ira fa morire gli insensati.

${ }^{52}$ In Rom., c. 16, lect. 2, nn. 1214-1217 (Rm 16,17: Vi raccomando poi, fratelli, di guardarvi da coloro che provocano divisioni e ostacoli contro l'insegnamento che avete appreso: tenetevi lontani da loro - Rogo autem vos, fratres, ut abservetis eos qui dissensiones et offendicula, praeter doctrinam quam vos didicistis, faciunt, et declinate ab illis): Perché coloro che l'Apostolo voleva fossero evitati, gli ingannatori, andavano fraudolentemente sotto la specie della pietà, secondo ciò che in Mt 7,15: Vengono a voi in vesti di pecore, ma dentro sono lupi rapaci; perciò, in primo luogo, induce i Romani (eos) ad avere la cautela dicendo: Vi prego poi, fratelli, di osservare coloro che fanno dissensi e ostacoli davanti alla dottrina che voi avete imparato. Qui, in primo luogo, bisogna considerare che "osservare" è nient'altro che "considerare diligentemente", il che talvolta si riferisce al bene, talvolta al male. Al male poi si riferisce appunto quando uno considera diligentemente la condizione e il progresso di qualcuno per nuocergli, secondo ciò che in Sal 37/36/,12: Il peccatore osservava il giusto e strideva sopra di lui $i$ suoi denti. Ed in Lc 14,1 è detto: Ed essi lo osservavano. Al bene, invece, si riferisce - in un modo - quando uno considera i precetti di Dio per osservarli (ad faciendum). Es 23,21: Osserva dunque ed ascolta la sua voce. In un altro modo, quando considera diligentemente i buoni per imitarli, secondo ciò che in Fil 3,17: Siate miei imitatori, fratelli, e osservate coloro che si comportano così come avete l'esempio nostro. In terzo modo, i cattivi vengono osservati per guardarsi da loro (ad cavendum), e così s'intende qui. Erano infatti alcuni Giudei convertiti alla fede, che predicavano che le prescrizioni della Legge devono essere osservate; e perciò, in primo luogo, nella Chiesa sorgevano i dissensi e le sette, quando alcuni aderivano ai loro errori, alcuni invece persistevano nella vera fede. Gal 5,20: Dissensi, sette, ecc. In secondo luogo, sorgevano offese e scandali di cui sopra, Rm 14 (nn. 1081, 1131), perché alcuni giudicavano gli altri, e gli uni disprezzavano gli altri che facevano dissensi ed ostacoli. Is 57,14 : Rimuovete gli ostacoli di mezzo al mio popolo. L'Apostolo poi dice: davanti alla dottrina che avete imparato dai veri Apostoli di Cristo, per mostrare che i dissensi di tale tipo e gli scandali provenivano dalla falsità della dottrina. Gal 1,9: Se qualcuno vi annuncia il vangelo diverso da quello che avete ricevuto, sia anàtema! In secondo luogo, l'Apostolo ammonisce che siano evitati 
cioè eresie. 2 Pt 2,1: Introdurranno sette della perdizione, ecc. Ed ivi /2 Pt 2,10/: Non temono d'introdurre le sette blasfeme. Dopo queste segue l'invidia, quando prosperano quelli che rivaleggiano. Gb 5,2: L'invidia uccide il piccolo, ecc. Da queste seguono gli omicidi nel cuore e con le opere (cordis et operis). 1 Gv 3,15: Chi odia il suo fratello è omicida ${ }^{53}$.

Quanto ai vizi che riguardano le cose necessarie alla vita - l'Angelico torna alla sua prima grande divisione - l'Apostolo ne pone due:

Uno, quanto al bere; perciò dice: ubriachezze, cioè assidue, Lc 21,34: State attenti che $i$ vostri cuori non si appesantiscano in crapula ed ubriachezza, ecc (e in cure di questa vita - W.D. con Vlg). L'altro invece, quanto al cibo, e quanto a ciò dice: gozzoviglie, $\mathrm{Rm} 13,13\left({ }^{54}\right)$ : Non nelle gozzoviglie e ubriachezze ${ }^{55}$.

E adesso possiamo passare a Gal 5,22-23a.

i conosciuti /falsi apostoli/ dicendo: e scostatevi da loro, cioè fuggite la loro dottrina e compagnia. Sal 119/118/,115: Scostatevi da me, maligni, e cercherò (scrutabor) $i$ comandamenti del mio Dio. Sal 37/36/,12: BG e BT: Il malvagio trama contro il giusto, contro di lui digrigna $i$ denti. - Es 23,21: BG: Abbi rispetto della sua presenza, da' ascolto alla sua voce; BT: Rispettalo ed ascolta la sua voce. - Fil 3,17: BG e BT: Fratelli, fatevi insieme miei imitatori e guardate quelli che si comportano secondo l'esempio che avete da noi. - Is 57,14: BG e BT: Rimuovete gli ostacoli sulla via del mio popolo. - Sal 119/118/,115: BG: Allontanatevi da me, o malvagi: voglio custodire i comandi del mio Dio; BT: Scostatevi da me, o malfattori, custodirò i comandamenti del mio Dio.

${ }^{53}$ In Gal., c. 5, lect. 5, nn. 325; vedi anche S.Th., II-II, q. 11, a. 1, ad 3; q. 37, a. 1; q. 38, a. 1; q. 41 , a. 1 ; q. 48 , a. 1 ; q. 158 , a. 3 . Contesa è l'attacco alla verità con confidenza nel clamore (Contentio est impugnatio veritatis cum confidentia clamoris): S. Ambrogio in Glossa ordin. super Rom. 1,29 (VI, 6 A); Pietro Lombardo, Glossa super Rom. 1,29 (PL 191, 1335) (nota - W.D.) Pr 20,3: BG: È una gloria evitare le contese; BT ha: per l'uomo. - Gc 1,20: BG: Infatti l'ira dell'uomo non compie ciò che è giusto davanti a Dio; BT: non compie la giustizia di Dio. - Ef 4,26: BG e BT: Non tramonti il sole sopra la vostra ira. - Pr 10,12: BG: L'odio suscita litigi; BT: conduce a. - Rm 16,17: Vi raccomando poi, fratelli, di guardarvi da coloro che provocano divisioni e ostacoli contro l'insegnamento che avete appreso: tenetevi lontani da loro; BT: Vi prego, fratelli, ... contese e scandali a dispetto della dottrina... Evitateli. - $2 \mathrm{Pt} 2,1$ : Introdurranno fazioni che portano alla rovina; BT: Introducono tra di voi eresie deleterie. - 2 Pt 2,10: Non temono d'insultare gli esseri gloriosi decaduti; con la nota agli "esseri gloriosi"; similmente BT, la quale nell'edizione del 1983, nella nota a questo versetto diceva che la Vlg ha: Non temono d'introdurre le sette. - Gb 5,2: L'invidia fa morire lo sciocco; BT: L'ira fa morire gli insensati.

${ }^{54}$ In Rom., c. 13, lect. 3, nn. 1074-1075 (Rm 13,13: Non in comessationibus et ebrietatibus): Qui l'Apostolo, in primo luogo, espone in che modo bisogna gettare via le opere delle tenebre, che sono opere dei peccati, e ne enumera alcune. In primo luogo pone quelle che si riferiscono alla corruzione del concupiscibile, la cui corruzione è l'intemperanza che riguarda i godimenti del tatto ed il cibo. Perciò prima esclude l'intemperanza nel cibo, quando dice: non nelle gozzoviglie. Le gozzoviglie consistono nel mangiare cibi superflui ed eccessivamente accurati. Pr 23,20: Non vogliate far parte (esse in) dei conviti dei peccatori né delle gozzoviglie di coloro che godono di cibarsi di carni. Che ciò può essere peccato mortale /risulta/ dal fatto che secondo la Legge per questa colpa qualcuno è stato condannato alla morte. Infatti, in Dt 21, 20s è detto del figlio impudente: Si dà (vacat) alle gozzoviglie e alla lussuria ed ai conviti; il popolo lo lapiderà. Non si dice però che si dà alle gozzoviglie ed ai conviti di uno che mangia magnificamente secondo lo stato 


\section{I frutti dello Spirito Santo}

D.S. Dockery afferma che "Gal 5,22-23 rappresenta il testo principale nel quale viene delineato il « frutto dello Spirito »" ed "offre una forma positiva della vita nello Spirito" $" 56$.

In Gal 5,22-23a, che è profondamente influenzato "da virtù tipicamente cristiane" 57 , leggiamo: Il frutto dello Spirito invece è amore, gioia, pace, magnanimità, benevolenza, bontà, fedeltà, mitezza, dominio di sé - Fructus autem spiritus est charitas, gaudium, pax, patientia, benignitas, bonitas, longanimitas, mansuetudo, fides, modestia, continentia, $\operatorname{castitas}\left({ }^{58}\right)$.

U. Vanni così commenta questo testo: "Alle opere della carne viene contrapposto il frutto dello Spirito: amore del prossimo; gioia: « che ha una base religiosa, fondata nella consapevole relazione di amicizia con Dio » (Burton, 31459); longanimità: nel senso di pazienza, sopportazione; fiducia: qui, probabilmente, senso di fiducia che uno ispira, attendibilità; padronanza di sé: il termine è usato

della sua dignità, come è detto in Est 2,18 che Assuero ordinò di preparare un magnifico convito per festeggiare con magnificenza principale il matrimonio con Ester. Lo si dice invece quando uno lo fa oltre la decenza del suo stato, e particolarmente se a ciò si volge principalmente la sua cura, come coloro di cui è detto in Rm 16,18: /Gli uomini/ di tale tipo non servono Cristo Signore, ma il loro ventre; e Fil3,19: Il loro dio è il ventre. Poi l'Apostolo esclude l'intemperanza nel bere, quando soggiunge: e ubriachezze, che consistono nell'eccesso del bere (ad superfluitatem potus) oltre la misura posta dalla ragione umana. Sir 31,35: Il vino è stato creato per la gioia e non per l'ubriachezza. E bisogna considerare che l'ubriachezza dal suo genere è peccato mortale, perché quando l'uomo si ubriaca di proposito, sembra che lui preferisca il godimento del vino all'integrità della ragione. Perciò è detto in Is 5,22: Guai a voi che siete potenti nel bere vino, e uomini forti nel mescere /bevandel per l'ubriachezza. Se invece uno si ubriacasse oltre la sua intenzione, non di proposito, per es. perché ignora la forza del vino o perché non pensava di poter ubriacarsi con una tale quantità della bevanda, non è peccato mortale, perché non si è ubriacato appositamente (per $s e$ ), ma accidentalmente (per accidens), cioè oltre l'intenzione, il che però non può riferirsi a coloro che si ubriacano frequentemente. E perciò Agostino, nel Sermone sul Purgatorio (PL 39, 1946 nota W.D.), dice che l'ubriachezza, se è assidua, è peccato mortale. Perciò qui l'Apostolo significativamente dice in plurale: Non nelle gozzoviglie e ubriachezze. Pr 23,20: BG e BT: Non essere fra quelli che s'inebriano di vino né fra coloro che sono ingordi di carne. - Dt 21, 20s: BG: È un ingordo e un ubriacone. Allora tutti gli uomini della sua città lo lapideranno; BT: Si dà alla lussuria e all'ubriachezza. Allora gli uomini di quella città ecc. - Sir 31,27/35/: BG e BT non hanno: e non per l'ubriachezza. - Is 5,22: Guai a coloro che sono gagliardi nel bere vino, valorosi nel mescere bevande inebrianti; BT: Guai a coloro che sono eroi nel bere vino, e spavaldi ecc.

${ }^{55}$ In Gal., c. 5, lect. 5, nn. 326; vedi anche S.Th., II-II, q. 150, a. 2; In 1 Cor., c. 5, lect. 3, n. 258. Lc 21,34: BG: State attenti a voi stessi, che i vostri cuori non si appesantiscano in dissipazioni, ubriachezze e affanni della vita; BT come Vlg.

${ }^{56}$ D.S. Dockery, Frutto dello Spirito, in G.F. Hawthorne - R.P. Martin - D.G. Reid (a cura di), Dizionario di Paolo e delle sue lettere, op. cit., pp. 652-656, qui p. 653.

${ }^{57}$ C.G. Kruse, Virtù e vizi, op. cit., p. 1607.

${ }^{58}$ BG nella nota dice che la Vlg, il codice di Beza e altri aggiungono: castità.

${ }^{59}$ E.W. De Burton, A Critical and Exegetical Commentary on the Epistle to the Galatians, op. cit., p. 307. 
anche nel greco classico nel senso di dominio di sé, dominio dei propri impulsi. Tutto questo proviene dallo Spirito per un suo influsso attivo" ${ }^{\circ}$.

San Tommaso dedica a Gal 5,22-23a un'intera lezione.

Prima di passare all'esame del testo in cui l'Apostolo "enumera i beni spirituali che chiama frutti" ${ }^{1}$, l'Aquinate vuole risolvere una questione che riguarda il linguaggio. Introducendo nella questione, dice:

Frutto si dice ciò di cui fruiamo, ma non dobbiamo fruire dei nostri atti, ma solo di Dio; dunque gli atti di tale tipo, che l'Apostolo qui enumera, non dobbiamo dire frutti. Anche la Glossa dice che di tale tipo opere dello spirito "sono di per sé appetibili"; ciò, invece, che di per sé è appetibile non va riferito ad un'altra cosa, dunque le virtù e le loro opere non vanno riferite alla beatitudine ${ }^{62}$.

Ed ecco, molto ampia ed esauriente, la risposta dell'Angelico; e, in primo luogo, riguardo alla questione del frutto:

Frutto si dice duplicemente, cioè come acquisito, ad es. dall'albero o dalla fatica, Sap 3,15: Glorioso è il frutto dei buoni lavori; e come prodotto, così come il frutto è prodotto dall'albero. Mt 7,18: Un albero buono non può produrre frutti cattivi. Le opere dello Spirito, invece, si dicono frutti non come ottenuti (adepti), ossia acquisiti, ma come prodotti; il frutto poi, che è ottenuto, ha ragione del fine ultimo, non invece il frutto prodotto. Ciononostante il frutto così ricevuto (acceptus) comporta però due cose, cioè che sia l'ultimo del producente, così come l'ultimo che è prodotto dall'albero è il suo frutto, e che sia soave e dilettabile. Ct 2,3: Il suo frutto è dolce al mio palato. Così dunque le opere delle virtù e dello spirito, sono ciò che è l'ultimo in noi. Lo Spirito Santo, infatti, è in noi mediante la grazia, per mezzo della quale acquisiamo l'abito delle virtù, e da ciò siamo in grado (potentes sumus) di operare secondo la virtù. /Queste opere/ sono anche dilettabili, e sono anche fruttuose. Rm 6,22: Avete il vostro frutto nella santificazione $\left({ }^{63}\right)$, cioè nelle opere santificate, e perciò si dicono frutto. Si dicono anche fiori rispetto alla futura beatitudine, perché come dai fiori si riceve la speranza dei frutti, così dalle opere delle virtù si ha la speranza della vita eterna e della beatitudine. E come nel fiore c'è qualche inizio (inchoatio) del frutto,

${ }^{60}$ U. Vanni, Lettera ai Galati, in P. Rossano (a cura di), Lettere di san Paolo, op. cit., p. 257.

${ }^{61}$ In Gal., c. 5, lect. 6, n. 328.

${ }^{62}$ In Gal., c. 5, lect. 6, n. 328; vedi anche S.Th., I-II, q. 11, a. 3 (Utrum fruitio sit tantum ultimi finis; resp. - sì), ob. 2 (presa da Gal 5,22) e ad 2. Glossa: Pietro Lombardo, Glossa super Gal. 5,22: col. 160 (PL 192 - compl. W.D.); vedi anche ID., Sent., lib. 1, d. 1, c. 3 (QR I, 19); cfr. Glossa interl., super Gal. 5,22 (VI, 87v); S. Ambrogio, In Gal., super 5,22 (PL 17, 389) (nota - W.D.).

${ }^{63}$ In Rom., c. 6, lect. 4, n. 514 (Rm 6,22: Raccogliete il frutto per la vostra santificazione Habetis fructum vestrum in sanctificationem): Qui l'Apostolo pone l'effetto della giustizia dicendo: Avete il vostro frutto nella santificazione, cioè la santificazione stessa, ossia l'esecuzione della santificazione per mezzo delle buone opere, è il vostro frutto, in quanto cioè questo vi diletta spiritualmente e santamente. Sir 24,17/23/: I miei fiori sono frutti di onore e onestà, ecc. Gal 5,22: Il frutto dello spirito è gioia, pace, ecc. Sir 24,17/23/: BG e BT: I miei fiori danno frutti di gloria e ricchezza. 
così nelle opere delle virtù c'è qualche inizio della beatitudine, che ci sarà allora quando saranno perfezionate la conoscenza e la carità ${ }^{64}$.

Questa spiegazione del nostro medievale magister in Sacra Pagina, il quale aveva davanti a sé il testo latino della Volgata, è attuale anche nei tempi odierni. R. Hensel, ad esempio, dopo aver accennato che Paolo considera le opere buone come frutto della fede, della giustizia (Fil 1,11; cfr. Eb 12,11; Gc 3,18), della luce $($ Ef 5,9$)$ operate da Dio, da Gesù Cristo o dallo Spirito Santo, e distingue chiaramente tra questo frutto e gli sforzi che fa l'uomo per il raggiungimento della salvezza, dice che in Paolo il concetto di frutto-ergon "è un concetto che proviene dal mondo tecnico-artigiano e indica ciò che l'uomo è capace di « produrre » coi propri sforzi e al propria abilità; karpós, invece, trae origine dalla sfera della vita e indica ciò che cresce per forza di cose (cfr. automáte = da sé di Mt 4,28), grazie all'energia vitale insita nella pianta o nel terreno; questa crescita è sintomo di vita, ma non è soggetta al potere dell'uomo. Paolo vuol dire in sostanza con questi concetti che non tarderanno a manifestarsi gli effetti, $\mathrm{i}$ « frutti », in colui che è stato inserito nel corpo di Cristo, nel quale opera lo Spirito di Dio, in colui che partecipa dei « doni » di questa comunanza; e sono appunto « frutti »- non «prodotti »- che vengono « come da se stessi ». Mentre i credenti in Cristo portano frutto per Dio, la potenza dei desideri peccaminosi produce frutti di morte (Rm $7,4 \mathrm{~s}){ }^{\prime 65}$.

Già nella spiegazione del concetto di frutto, l'Aquinate ha indicato alcuni elementi della risposta alla seconda obiezione, cioè quella presa dalla Glossa e riguardante la beatitudine, ma a lui ciò non basta, perciò prosegue la sua lezione:

Una cosa può essere detta appetibile di per sé in due modi, perché lì il di per sé (propter) può designare la causa formale o finale. Le opere delle virtù di per sé (propter se) sono appetibili formalmente, ma non finalmente, perché hanno in se stesse il godimento (delectatio). Infatti, una medicina dolce la si desidera di per sé formalmente, perché ha in sé donde sia appetibile, cioè la dolcezza, che tuttavia è desiderata per il fine (propter finem), ossia per la salute. Ma una medicina amara non è desiderata di per sé formalmente, perché non diletta a ragione della sua forma, ma tuttavia è desiderata per (propter) un'altra cosa, cioè per la salute che è il suo fine. Da ciò risulta la ragione per cui l'effetto della carne l'Apostolo chiama opere, i frutti dello Spirito invece chiama frutti ${ }^{66}$.

${ }^{64}$ In Gal., c. 5, lect. 6, n. 328. Sap 3,15: Vlg: Bonorum laborum gloriosus est fructus; BG: delle opere buone; $\mathrm{BT}$ : delle buone fatiche.

${ }^{65}$ R. Hensel, Frutto, in L. Coenen - E. Beyreuther - H. Bietenhard (a cura di), Dizionario dei concetti biblici del Nuovo Testamento, op. cit., pp. 728-730, qui pp. 729-730; vedi anche B. Gillièron, Lessico dei termini biblici, op. cit., voce: Frutto, pp. 94-95, qui p. 94; C. Spicq - X. Leon-Dufour, Frutto, in X. Leon-Dufour (dir.), Dizionario di teologia biblica, op. cit., coll. 430-433, qui coll. 432-433; X. Leon-Dufour, Dizionario del Nuovo Testamento, op. cit, voce: Frutto, pp. 266-267.

${ }^{66}$ In Gal., c. 5, lect. 6, n. 328. 
Ma anche questa spiegazione non basta, e san Tommaso continua:

Frutto si dice qualcosa finale e soave, prodotto da una cosa. Ciò infatti che è prodotto da una cosa fuori della sua natura non ha ragione di frutto, ma come di un altro germe. Le opere della carne, poi, e i peccati sono fuori della natura delle cose che Dio ha inserito nella nostra natura. Dio, infatti, ha inserito nella natura umana alcuni semi, cioè il naturale appetito del bene e della conoscenza, e ha aggiunto anche i doni della grazia. E perciò, perché le opere delle virtù sono naturalmente prodotte da questi /doni/, /queste opere/ si dicono frutti, non invece le opere della carne. E per questo l'Apostolo dice in $\mathrm{Rm}$ 6,21: Che frutto dunque avete avuto allora in quelle cose di cui adesso vi vergognate? $\left({ }^{67}\right)$. Da ciò che è stato detto risulta dunque che frutti dello spirito si dicono le opere delle virtù, e perché hanno in sé la soavità e la dolcezza, e perché sono qualche ultimo prodotto secondo la convenienza dei doni ${ }^{68}$.

Nella parte conclusiva di questa ampia introduzione alla sua esposizione, l'Aquinate dimostra la reciproca differenza tra dono, beatitudine, virtù e frutto $\left({ }^{69}\right)$ :

Nella virtù, infatti, c'è da considerare l'abito e l'atto. L'abito della virtù, però, perfeziona ad agire bene. E se appunto perfeziona ad operare bene in modo umano, si dice virtù. Se invece perfezione ad operare bene sopra il modo umano, si dice dono. Perciò il Filosofo sopra le virtù comuni pone alcune virtù eroiche, ad es. conoscere le cose invisibili di Dio sotto enigma avviene per modo umano: e questa conoscenza appartiene alla virtù della fede; ma conoscere queste cose perspicacemente e sopra il modo umano, appartiene al dono dell'intelletto. L'atto della virtù, infatti, o perfeziona: e così è beatitudine; o diletta: e così è frutto. $E$ di questi frutti si dice in Ap 22,2: $E$ da una parte e dall'altra del fiume, si trova un albero di vita che dà frutti dodici volte all'anno, ecc ${ }^{70}$.

${ }^{67}$ In Rom., c. 6, lect. 4, n. 510 (Rm 6,21: Quem ergo fructum habuistis tunc in illis, in quibus nunc erubescitis?): Qui l'Apostolo dimostra l'effetto del peccato. Ed esclude appunto un effetto, cioè l'effetto fruttuoso, quando dice: che frutto dunque avete avuto, quando cioè peccavate in quelle cose, cioè nei peccati. Le opere dei peccati, infatti, sono infruttuose, perché non aiutano l'uomo a conseguire la beatitudine. Is 59,6: Le loro opere sono opere inutili. Mi 2,1: Guai a voi che escogitate le cose inutili ed operate il male nei vostri giacigli. Aggiunge invece l'effetto degno di vergogna, dicendo: delle quali, ossia dei peccati, cioè dei quali adesso, cioè in stato della penitenza, vi vergognate, a motivo della loro bruttezza. Ger 31,19: Dopo che me l'hai dimostrato, mi sono battuto il petto, ho provato confusione e mi sono vergognato. Is 1,29: Vi vergognerete dei giardini, cioè /dei giardini/ della voluttà che avete scelto. Is 59,6: BG e BT: inique. - Mi 2,1: BG e BT: Guai a coloro che meditano l'iniquità e tramano il male sui loro giacigli.

${ }^{68}$ In Gal., c. 5, lect. 6, n. 328; per tutto questo n. 328, vedi anche S.Th., I-II, q. 70, a. 1 (Utrum fructus Spiritus Sancti quos Apostolus nominat ad Galatas 5, sint actus; resp. - sì) (così non c'è nessun dubbio che fructus spiritus della Volgata san Tommaso intende come frutti dello Spirito Santo - nota W.D.); a. 2 (Utrum fructus a beatitudinibus differant; resp. - sì); vedi anche In Isaiam, c. 11. Sap 3,15: Vlg: Bonorum laborum gloriosus est fructus; BG: delle opere buone; BT: delle buone fatiche.

${ }^{69}$ Cfr. D.S. Dockery, Frutto dello Spirito, op. cit., p. 654.

${ }^{70}$ In Gal., c. 5, lect. 6, n. 329; vedi anche S.Th., I-II, q. 68, a. 1 (Utrum dona differant a virtutibus; resp. - sì); q. 70, aa. 1-2; II-II, q. 52, a. 4, ad 3; In III Sent., d. 34, q. 1, a. 1; In Isaiam, c. 11. Filosofo: Aristotele, Etica, lib. 7, 1, 1 (1145 a 20) (nota - W.D.). 
Solo dopo aver chiarito tutte queste cose, l'Angelico passa all'esposizione del testo di Gal 5,22-23a. I frutti dello Spirito enumerati in questo testo, "che spuntano nell' anima dalla seminagione della grazia spirituale" "71, il nostro teologo-esegeta li divide in due gruppi: quelli che perfezionano interiormente e quelli che perfezionano esteriormente.

Per D.S. Dockery, che prende in esame tutti i frutti dello Spirito elencati in Gal 5,22-23, "è molto importante che l'amore si trovi in cima alla lista. Quest'enfasi è presente anche altrove in Paolo. L'amore caratterizza Dio e quando viene realizzato da uomini e donne, questo adempie la Legge (Rm 13,10). Esso rappresenta un'azione di donazione di sé per il bene degli altri, non necessariamente un'emozione" 72 .

San Tommaso, parlando dei frutti che perfezionano interiormente, prima introduce in generale:

L'uomo viene perfezionato e diretto interiormente sia circa i beni che circa i mali. 2 Cor 6,7(73): Per mezzo delle armi della giustizia a destra e a sinistra ${ }^{74}$.

Circa i beni - continua l'Aquinate, affrontando il primo punto - i frutti dello Spirito perfezionano innanzitutto proprio nel cuore mediante l'amore:

Infatti, come tra i moti naturali il primo è l'inclinazione dell'appetito naturale al suo fine, così il primo dei moti interiori è l'inclinazione al bene, che si dice amore, e perciò il primo frutto è la carità, Rm 5,5: La carità di Dio è stata diffusa nei nostri cuori, ecc $\left({ }^{75}\right)$. E dalla carità vengono perfezionate le altre /virtù/, e perciò l'Apostolo

${ }^{71}$ In Gal., c. 5, lect. 6, n. 330.

${ }^{72}$ D.S. Dockery, Frutto dello Spirito, op. cit., p. 655.

${ }^{73}$ In 2 Cor., c. 6, lect. 2, n. 223: Qui l'Apostolo fa vedere in che modo /quelle virtù/ (vedi 2 Cor 6,6; In 2 Cor., nn. 216-222 - nota W.D.) si hanno nell'operazione dei beni e dei mali, tra i beni e i mali, nelle prosperità e nelle avversità; e questo appartiene alla virtù della giustizia. (...) Prima, dunque, l'Apostolo dice che ci presentiamo come ministri di Dio con molta pazienza (vedi 2 Cor 6,4; In 2 Cor., nn. 210-211 - nota W.D.). E c'è più di questo, per mezzo delle armi della giustizia. Qui bisogna sapere che la giustizia ordina l'uomo e lo fa tenere il suo posto, a destra, cioè nelle prosperità, perché non si elevi, e a sinistra, cioè nelle avversità, perché non si abbassi. Fil 4,12: Sono allenato a tutto, ecc., all'abbondanza, ecc (e all'indigenza - W.D. con BG).

${ }^{74}$ In Gal., c. 5, lect. 6, n. 330.

${ }^{75}$ In Rom., c. 5, lect. 1, n. 392: L'Apostolo dunque, in primo luogo, dice: Che la speranza non delude (Rm 5,5; In Rom., n. 390 - nota W.D.) lo possiamo sapere dal fatto che la carità di Dio è stata diffusa nei nostri cuori per mezzo dello Spirito Santo che ci è stato dato. La carità di Dio, poi, può essere intesa duplicemente. Un modo: come carità con cui Dio ci ama, Ger 31,3: Ti ho amato di carità perpetua; l'altro modo: possiamo dire carità di Dio quella /carità/ con cui noi amiamo Dio, Rm 8,38s: Sono certo che neanche la vita può separarci dalla carità di Dio. Entrambe le carità di Dio, poi, vengono diffuse nei nostri cuori mediante lo Spirito Santo che ci è stato dato. Infatti, dare a noi lo Spirito Santo, il quale è Amore del Padre e del Figlio, significa condurci alla partecipazione dell'amore che è lo Spirito Santo. Grazie a questa partecipazione, appunto, noi diventiamo efficacemente amatori di Dio. E che noi lo amiamo è segno del fatto che egli ci ama. Pr 8,17: Io amo coloro che mi amano. $1 \mathrm{Gv}$ 4,10: Non siamo stati noi ad amare Dio per primo, ma 
dice in Col 3,14: Al di sopra di tutte /le virtù/ abbiate la carità, ecc $\left({ }^{76}\right)$. Il fine ultimo, invece, con cui l'uomo è perfezionato interiormente, è il gaudio che proviene dalla presenza della cosa amata. Chi poi ha la carità, ha già ciò che ama. $1 \mathrm{Gv} 4,16$ : Chi rimane nell'amore rimane in Dio e Dio in lui. E da ciò sorge il gaudio. Fil 4,4: Gioite sempre nel Signore $\left({ }^{77}\right)$, ecc ${ }^{78}$.

Per quanto riguarda la gioia, D.S. Dockery osserva: "Anche se Paolo spesso esorta i credenti a gioire nel Signore (cfr. Fil 3,1;4,4), nella lista del frutto dello Spirito si trova l'unica occorrenza della parola gioia in Galati. Anche nelle prove

egli ci ha amati per primo. Diciamo poi che la carità, con cui /Dio/ ci ama, è stata diffusa nei nostri cuori, perché è chiaro che essa è nei nostri cuori mediante il dono dello Spirito Santo in noi impresso. Diciamo poi che la carità, con cui noi amiamo Dio, è stata diffusa nei nostri cuori, perché cioè si estende, perfezionandoli, a tutti i costumi ed atti dell'anima. 1 Cor 13,4: La carità è paziente, è benigna, ecc. Vedi anche C.G., III, c. 151. Ger 31,3: BG e BT: di amore eterno.

${ }^{76}$ In Col., c. 3, lect. 3, n. 163: Al di sopra di tutte /le virtù/ (vedi la divisione del testo, In Col., n. 162 - nota W.D.) rivestite la carità, la quale fra tutte le predette virtù (vedi Col 3,12-13; In Col., nn. 157-161) è la più grande, come è detto in 1 Cor 13,13. Al di sopra di tutte, cioè più di tutte, perché è il fine di tutte le virtù. $1 \mathrm{Tm}$ 1,5: Il fine del precetto è la carità. Oppure: al di sopra di tutte /le virtù/ dobbiamo avere la carità, perché è al di sopra di tutte le altre. 1 Cor 12,31: Io vi mostrerò una via più eccellente di tutte. E ciò perché senza di questa le altre non valgono nulla. E questa carità è raffigurata dalla tunica senza cuciture, Gv 19,23. E la ragione per cui bisogna averla è indicata dalle parole: è il vincolo. Secondo la Glossa, l'uomo è perfezionato per mezzo di tutte le virtù, ma la carità le connette a vicenda e le rende perseveranti, e perciò è detta vincolo. Oppure dalla sua natura è il vincolo, perché è l'amore che unisce l'amato all'amante. Os 11,4: Io ti traevo con legami di bontà, con vincoli d'amore, ecc. Ma l'Apostolo aggiunge: della perfezione, perché uno è perfetto quando aderisce al fine ultimo, cioè a Dio, il che fa la carità. Vedi anche S.Th., I-II, q. 65, aa. 3-5; q. 66, a. 6; II-II, q. 4, a. 7, ad 4; q. 23, aa. 6-8; q. 184, a. 1; De Veritate, q. 14, a. 5; In II Sent., d. 26, a. 4, ad 5; In III Sent., d. 23, q. 3, a. 1, qc. 1; d. 27, q. 2, a. 4, qc. 3; De Malo, q. 7, a. 2; De Caritate, a. 3; De Perfect. vitae spirit., cc. 1ss; Contra retrahentes hom. ab ingressu religionis, c. 6; Quodlib., IV, q. 10, a. 1; S.Th., II-II, q. 124, a. 3; Quodlib., III, q. 6, a. 3; IV, q. 12, a. 2, ad 2. 1 Cor 12,31: Vlg: Et adhuc excellentiorem viam vobis demonstro; BG: la via più sublime; BT: una via ancora più perfetta. Glossa: Glossa ordin. (VI, 30 B); Pietro Lombardo, Glossae (PL 191, 1520) (nota - W.D.).

${ }^{77}$ In Philipp., c. 4, lect. 1, n. 153: A chiunque vuole progredire è necessario che abbia il gaudio spirituale. Pr 17,22: Un animo gaudente fa florida l'età, ecc. (...) La prima /condizione del vero gaudio/ è che dev'essere retto, il che è quando è del bene proprio dell'uomo, che non è qualcosa di creato, ma Dio, Sal 73/72/,28: Il mio bene è aderire a Dio, ecc. Allora, dunque, /il gaudio/ è retto, quando è nel Signore. Perciò l'Apostolo dice: nel Signore. Ne 8,10: La gioia del Signore è la vostra forza. /Il gaudio dev'essere/ anche continuo; perciò l'Apostolo dice: sempre. 1 Ts 5,16: Gioite sempre. Pr 17,22: Vlg: Animus gaudens floridam aetatem facit; BG: Un cuore lieto fa bene al corpo, uno spirito depresso inaridisce le ossa; BT: La gioia del cuore fa bene alla salute, ecc. come BG. - Sal 73/72/,28: Vlg: Mihi adhaerere Deo bonum est; BG e BT: Per me, il mio bene è stare vicino a Dio. 1 Ts 5,16; In 1 Thess., c. 5, lect. 2, n. 129 (Siate sempre lieti - Gudete semper): Qui l'Apostolo dimostra in che modo /i Tessalonicesi/ devono comportarsi verso Dio, (...). In primo luogo, devono gioire di Lui, perciò dice: gioite sempre, cioè di Dio, perché, qualunque male venga, è incomparabile al bene che è Dio. E perciò nessun male lo interrompa; per questo dice: gioite sempre.

${ }^{78}$ In Gal., c. 5, lect. 6, n. 330. 
i credenti possono conservare la gioia nel Signore, che impedisce loro di cedere alla totale disperazione. Mentre l'amore è chiaramente l'attributo di Dio, lo stesso non si può dire, in modo esplicito, della gioia; ma il suo carattere divino è chiaro in altri contesti (per esempio, Rm 14,17)"'79.

L'Angelico, continuando la sua lezione, dice che questo gaudio deve essere perfetto, perciò si esigono due cose:

La prima, che la cosa amata, a motivo della sua perfezione, sia sufficiente all'amante. E quanto a ciò l'Apostolo dice: pace. Allora infatti l'amante ha la pace, quando sufficientemente possiede la cosa amata. Ct 8,10: Così io sono ai suoi occhi come colei che procura pace, ecc. La seconda cosa /che si esige/ è che ci sia la perfetta fruizione della cosa amata, il che similmente si ha per mezzo della pace, perché - qualunque cosa sopravvenga - se uno perfettamente fruisce della cosa amata, ad es. di Dio, non può essere impedito nella sua fruizione. Sal 119/118/,165: Grande pace per chi ama la tua legge, nel suo cammino non trova inciampo. Così dunque l'Apostolo dice gaudio la fruizione della carità, ma pace la perfezione della carità. E per mezzo di esse l'uomo viene perfezionato quanto ai beni ${ }^{80}$.

"L'esistenza cristiana genuina - afferma D.S. Dockery - dimostra la pace di Dio, in quanto i credenti sono in pace con Dio $(\mathrm{Rm} 5,1)$. (...) La pace è la serenità concessa ai credenti per sostenerli. H.D. Betz suggerisce che questa triplice struttura iniziale (amore, gioia, pace) riveli un importante elemento dell'etica paolina: «Non ci si può aspettare semplicemente di agire in modo responsabile, dal punto di vista etico; è necessario innanzitutto essere abilitati, potenziati e motivati prima di poter agire» (Betz 28781)"82.

San Tommaso, affrontando in seguito il secondo punto, afferma che anche circa i mali lo Spirito Santo perfeziona e ordina i fedeli:

Primo, contro il male che perturba la pace, la quale viene perturbata per mezzo delle avversità. Ma a ciò lo Spirito Santo perfeziona per mezzo della pazienza, la quale fa

${ }^{79}$ D.S. Dockery, Frutto dello Spirito, op. cit., p. 655. Anche san Tommaso, commentando Rm 14,17, indica questo carattere divino della gioia; In Rom., c. 14, lect. 2, n. 1129: L'Apostolo esprime la causa di questo gaudio, dicendo: nello Spirito Santo. Ed è mediante lo Spirito Santo, infatti, che la carità di Dio viene diffusa in noi, come è detto in $\mathrm{Rm}$ 5,5 (l'interpretazione di Rm 5,5, vedi sopra, In Rom., c. 5, lect. 1, n. 392 - nota W.D.). Il commento di Fil 3,1: Siate lieto nel Signore gaudete in Domino (BT: Gioite nel Signore), In Philipp., c. 3, lect. 1, n. 100: Gioite nel Signore soltanto, non nell'osservanza della Legge. Ab 3,18: Io invece gioirò nel Signore, ed esulterò in Dio, mio Salvatore, ecc. (qui la Volgata ha molto più forte: in Deo Iesu meo, perciò sembra che san Tommaso abbia scelto questo testo e non il parallelo Fil 4,4 - nota W.D.).

${ }^{80}$ In Gal., c. 5, lect. 6, n. 330; per tutto il n. 330, vedi anche S.Th., I-II, q. 70, a. 3 (Utrum fructus /Spiritus Sancti/ convenienter enumeretur ab Apostolo /Gal 5,22s/; resp. - sì); II-II, q. 28, a. 1 (Utrum gaudium sit effectus caritatis in nobis; resp. - sì); a. 4 (Utrum gaudium sit virtus; resp. - no); De Malo, q. 11, a. 2, ad 1; De Caritate, q. un., a. 2, ad 12.

${ }^{81}$ H.D. Betz, Galatians, Herm, Fortress, Philadelphia 1979, p. 287.

${ }^{82}$ D.S. Dockery, Frutto dello Spirito, op. cit., p. 655. 
sopportare pazientemente le avversità, e perciò l'Apostolo dice: pazienza. Lc 21,19: Nella vostra pazienza possiederete le vostre anime. Gc 1,4: La pazienza produce un'opera perfetta. Secondo, contro il male che impedisce il gaudio c'è il rinvio della cosa amata, al quale lo Spirito oppone la longanimità, la quale non viene infranta dall'attesa. E quanto a ciò l'Apostolo dice: longanimità. Ab 2,3: Se indugia, attendila, ecc (perché certo verrà e non tarderà - W.D. con BG). 2 Cor 6,6: Con longanimità, ecc( ${ }^{83}$; vedi anche $\mathrm{Col} 1,11^{84}$; Eb 6,15 ${ }^{85}$ - nota W.D.). E per questo il Signore dice in Mt 10,22: Chi persevererà sino alla fine, ecc ${ }^{86}$.

Secondo D.S. Dockery, "la pazienza rappresenta un valore positivo, che abbraccia fermezza e capacità di resistenza. La pazienza, o la tolleranza, è una qualità di Dio (Sal 103,8) e deve essere riprodotta nel popolo di Dio (1 Cor 13,4; Ef 4,2; Col 1,11; 3,12). Benevolenza. Lo Spirito Santo produce nei credenti l'attitu-

${ }^{83}$ In 2 Cor., c. 6, lect. 2, n. 219: (2 Cor 6,6: con magnanimità - in longanimitate): Qui l'Apostolo pone la virtù della speranza. Perciò dice: con longanimità, il che appartiene alla perfezione della speranza. La longanimità infatti non è nient'altro che il fatto che uno dalla speranza aspetta pazientemente una cosa importante (arduum) a lungo rinviata, e la /aspetta/ dallo Spirito Santo. Gal 5,22: Il frutto dello Spirito è carità, ecc., longanimità, ecc. Col 1,11: Con ogni pazienza e longanimità.

${ }^{84}$ In Col., c. 1, lect. 3, n. 22 (Col 1,11: In omni patientia et longanimitate, cum gaudio; BG: per essere perseveranti e magnanimi in tutto; BT: in ogni pazienza e costanza): Quando l'Apostolo dice: con ogni pazienza, ecc., chiede a loro (ai Colossesi) di sopportare le avversità. Alcuni infatti vengono meno o a motivo delle difficoltà delle cose avverse, e perciò bisogna avere la pazienza. Lc 21,19: Nella vostra pazienza possiederete le vostre anime. Oppure a motivo del rinvio del premio. E perciò dice: e longanimità, la quale fa sopportare /il rimando/ della cosa promessa. Ab 2,3: Se indugia, attendila, ecc. Eb 6,15: Aspettando con longanimità, ottenne ciò che gli era promesso. Ma alcuni evitano queste due cose, però con tristezza. Contro ciò dice: con gaudio. Gc 1,2: Considerate perfetta letizia, fratelli, quando subite ogni sorta di prove, ecc (sapendo che la vostra fede, messa alla prova, produce pazienza - W.D. con BG). Lc 21,19: BG e BT: Con la vostra perseveranza salverete la vostra vita.

${ }^{85}$ In Hebr., c. 6, lect. 4, n. 316 (Eb 6,15: Longanimiter ferens adeptus est repromissionem; BG: Con la sua costanza ottenne ecc., BT: E poiché così pazientemente aspettava, ottenne ecc.): E vi fu l'effetto della promessa, perché aspettando con longanimità, ottenne ciò che gli era promesso. La longanimità è non solo nel fare qualcosa di grande, ma anche nell'aspettare a lungo. Abramo, poi, ebbe la promessa, quando non possedeva neppure un pezzo della terra /promessa/, come è detto in At 7,5. E fino alla vecchiaia non ebbe la prole, e tuttavia non venne meno nella speranza. Gc 5,10: Prendete, fratelli miei, come esempio di sopportazione e di longanimità, di fatica e di pazienza i profeti, ecc. Is 51,2: Guardate ad Abramo, vostro padre, ecc. Gc 5,10: nel testo: Accipite exemplum, fratres mei, mali exitus, et longanimitatis, et laboris, et patientiae propheras; Vlg: Exemplum accipite, fratres, laboris et patientiae propheras; BG: ... a modello di sopportazione e di costanza i profeti (l'ed. BG del 1991 ha qui: di sopportazione e di pazienza) BT: di perseveranza e di pazienza.

${ }^{86}$ In Gal., c. 5, lect. 6, n. 331; vedi anche S.Th., II-II, q. 136, a. 1 (Utrum patientia sit virtus; resp. - sì); a. 5 (Utrum patientia sit idem quod longanimitas; resp. - no); q. 157, a. 2 (Utrum tam clementia quam mansuetudo sit virtus; resp. - sì); In 2 Cor., c. 6, lect. 2, n. 219. Lc 21,19: BG e BT: Con la vostra perseveranza salverete la vostra vita. - Gc 1,4: Vlg: Patientia opus perfectum habet; BG: La pazienza completi l'opera sua in voi; BT: La perseveranza deve essere un'opera perfetta. 
dine alla dolcezza, o benevolenza, dinamizzando l'amore. La bontà è una qualità dell'azione benevola di Dio e delle sue azioni verso i peccatori $(\operatorname{Rm} 2,4$; Ef 2,7; Tt 3,4). I cristiani non trovano migliore modalità per proseguire nella benevolenza di Dio che nel mostrare agli altri la benevolenza di Dio (Ef 4,32)" ${ }^{\prime \prime 7}$.

L'Aquinate, passando ai frutti dello Spirito che perfezionano l'uomo quanto alle cose esteriori, introduce in generale, facendo vedere anche qui che la vita esteriore dell'uomo non ha solo una dimensione personale, ma anche quella teologica e quella sociale:

Le cose esteriori dell'uomo, poi, sono o ciò che è accanto a lui (iuxta ipsum), o ciò che è sopra di lui, o ciò che è sotto di lui. Accanto a lui c'è il prossimo, sopra di lui c'è Dio, sotto di lui c'è la natura sensitiva e il corpo ${ }^{88}$.

Il quadro antropologico-esistenziale è molto chiaro, e le spiegazioni del nostro teologo-esegeta saranno molto ampie, esaurienti e pluridimensionali. Quanto al prossimo, in primo luogo, lo Spirito perfeziona cominciando dal cuore mediante la retta e buona volontà:

E quanto a ciò l'Apostolo dice: bontà, cioè la rettitudine e la dolcezza dell' animo. Se infatti l'uomo avesse buone tutte le altre potenze, non può essere detto buono l'uomo se non avesse la buona volontà, secondo la quale usa bene tutte le altre /potenze/. Ne è la ragione: perché buono si dice qualcosa di perfetto. La perfezione poi è duplice. La prima, cioè quella che è l'essere stesso della cosa. La seconda invece è la sua operazione: e questa è maggiore della prima. Si dice semplicemente perfetto ciò che si estende (pertingit) alla sua perfetta operazione, la quale è la sua seconda perfezione. Siccome dunque l'uomo mediante la volontà invade nell'atto (exeat in actum) di qualsiasi potenza, la retta volontà rende buono l'uso di tutte le potenze e, per conseguenza, buono l'uomo stesso. E di questo frutto si dice in Ef 5,9( $\left.{ }^{89}\right)$ : Il frutto della luce consiste in ogni bontà, $\mathrm{ecc}^{90}$.

${ }^{87}$ D.S. Dockery, Frutto dello Spirito, op. cit., p. 655.

${ }^{88}$ In Gal., c. 5, lect. 6, n. 332.

${ }^{89}$ In Eph., c. 5, lect. 4, n. 291: L'Apostolo dunque dice: Ho detto di comportarvi come figli della luce (Ef 5,8; vedi In Eph., n. 289 - nota W.D.), ora il frutto della luce sono le opere fruttifere e chiare. Sir 24,17/23/: I miei fiori sono frutti di onore, ecc (e di onestà - W.D. con Vlg). E ciò in ogni bontà, ecc. Qui bisogna sapere che ogni atto della virtù si riduce a tre cose. Bisogna infatti che l'agente sia ordinato verso di sé, verso il prossimo e verso Dio. Verso di sé, perché sia buono in se stesso, e perciò l'Apostolo dice: in ogni bontà. Sal 119/118/,66: Insegnami la bontà e la disciplina e la scienza, ecc. Anche, verso il prossimo mediante la giustizia. Perciò dice: e giustizia. Sal 119/118/ ,121: Ho fatto giudizio e giustizia, ecc. Verso Dio mediante la cognizione e la confessione della verità. E perciò dice: e verità. Zc 8,19: Amate la verità e la pace. Oppure /si può interpretare/ in un altro modo perché la bontà si riferisca al cuore, la giustizia all'opera, la verità alla bocca. Ef 4,25 e Zc 8,16: Dite la verità ciascuno con il suo prossimo. Sir 24,17/23/: BG e BT: I miei fiori danno frutti di gloria e ricchezza. - Sal 119/118/,66: BG: Insegnami il gusto del bene e la conoscenza; con la nota: "gusto del bene: BJ traduce: buon senso"; BT: Insegnami l'intelligenza e l'abilità. - Sal 119/118/,121: BG: Ho agito secondo giudizio e giustizia; BT: Faccio giudizio e giustizia.

${ }^{90}$ In Gal., c. 5, lect. 6, n. 332. 
Per quanto concerne la bontà, D.S. Dockery afferma che essa "include l'idea della generosità, ma si concentra sull'incidenza morale. Come termine inusuale, esso compare nella LXX e soltanto quattro volte nel NT. Fung ritiene che «la bontà è un'attitudine della generosa benevolenza verso gli altri, felice di realizzare molto di più di quanto viene richiesto dalla semplice giustizia » (Fung 268 ${ }^{91}$ ). Questa magnanime generosità è il contrario dell'invidia (Gal 5,21)"92.

In secondo luogo - andiamo insieme con l'Angelico - lo Spirito perfeziona l'uomo nell'operare, affinché condivida (communicet) le sue cose con il prossimo:

E quanto a ciò l'Apostolo dice: benignità, cioè la generosità nelle cose (largitas rerum). 2 Cor 9,7: Dio ama il datore gioioso $\left.{ }^{93}\right)$. La benignità infatti è detta bona igneitas, la quale fa l'uomo sciogliersi nel soccorrere le necessità degli altri. Sap 1,6: Benigno infatti è lo spirito della sapienza, ecc. Col 3,12( $\left.{ }^{94}\right)$ : Rivestitevi dunque, come eletti di Dio, santi e diletti, di sentimenti di misericordia, di benignità, ecc ${ }^{95}$.

I frutti dello Spirito - l'Angelico parla ancora del comportamento verso il prossimo - perfezionano l'uomo anche circa i mali inflitti dagli altri, affinché mansuetamente sopporti le molestie del prossimo:

${ }^{91}$ R.Y.K. Fung, The Epistle to the Galatians, NICNT, Eerdmans, Grand Rapids 1988, p. 268.

${ }^{92}$ D.S. Dockery, Frutto dello Spirito, op. cit., p. 655.

${ }^{93}$ In 2 Cor., c. 9, lect. 1, n. 332 (2 Cor 9,7: Dio ama chi dona con gioia - Hilarem datorem diligit Deus): Ogni rimuneratore rimunera ciò che è degno di rimunerazione. Lo sono appunto solo gli atti delle virtù. Negli atti delle virtù, poi, ci sono due cose, cioè la specie dell'atto e il modo dell'agire che è da parte dell'agente. (...) Ed anche se presso gli uomini, i quali non vedono se non ciò che appare, è sufficiente che uno operi l'atto della virtù secondo la specie dell'atto, per es. l'atto della giustizia, tuttavia presso Dio, il quale vede il cuore (2 Sam 16,7: L'uomo guarda l'apparenza, il Signore guarda il cuore - nota W.D.), non è sufficiente che si operi l'atto della virtù solo secondo la sua specie, ma anche che lo si operi secondo il debito modo, cioè con amore (delectabiliter) e con gioia. E perciò non il datore in quanto tale, ma il datore gioioso Dio ama, cioè approva e rimunera, e non il triste o il mormorante. Sal 100/99/,2: Servite il Signore nella gioia. Sir 35,11: In ogni dono mostra gioioso il tuo volto. $\mathrm{Rm} \mathrm{12,8:} \mathrm{Chi} \mathrm{fa} \mathrm{le} \mathrm{opere} \mathrm{di} \mathrm{misericordia,} \mathrm{lo} \mathrm{faccia} \mathrm{con}$ gioia. Vedi anche S.Th., I-II (e non II-II, come ha l'Ed. Marietti 1953, vol. 1, p. 515), q. 100, a. 9 (Utrum modus virtutis cadat sub praecepto legis; resp. - no), ad 3, dove c'è la risposta all'obiezione presa da Sal 100/99/,2 e da 2 Cor 9,7; Contra Impugnantes Dei cultum, c. 7.

${ }^{94}$ In Col., c. 3, lect. 3, n. 160: Verso tutti bisogna avere la benignità, la quale è come bona igneitas. Il fuoco infatti liquefa e fa evaporare l'umidità. Se in te c'è il fuoco buono (bonus ignis), esso liquefa qualsiasi cosa che tu abbia d'umidità, e la dissolve. Questo lo fa lo Spirito Santo. Sap 1,6: Benigno è lo spirito della sapienza. Ef 4,32: Siate benigni e misericordiosi gli uni verso gli altri, ecc. Nel cuore tu devi avere l'umiltà. Sir 3,18/20/: Quanto più sei grande, tanto più umiliati, ecc. Ef 4,32; In Eph., c. 4, lect. 10, n. 265: Qui l'Apostolo pone ciò che si riferisce alla novità contraria alle predette passioni (vedi Ef 4,31 ed In Eph., n. 264 - nota W.D.): contro l'amarezza la benignità. Perciò dice: siate benigni gli uni verso gli altri. Perché benigno è lo spirito della sapienza, ecc (Sap 1,6 Vlg - nota W.D.). Contro l'ira la misericordia; perciò dice: misericordiosi. Lc 6,36: Siate dunque misericordiosi, come è misericordioso il Padre vostro.

${ }^{95}$ In Gal., c. 5, lect. 6, n. 332. Sap 1,6: Vlg: Benignus est spiritus sapientiae; BG: La sapienza è uno spirito che ama l'uomo; BT: ... gli uomini. 
E quanto a ciò l'Apostolo dice: mansuetudine, Mt 11,29: Imparate da me, ecc (che sono mite e umile di cuore - W.D. con BG). Pr 3,34: Ai mansueti darà la grazia ${ }^{96}$.

Quanto invece a ciò che è sopra di noi, cioè quanto a Dio, lo Spirito ci ordina mediante la fede:

Perciò l'Apostolo dice: fede, che è una cognizione certa delle cose invisibili. Gen 15,6: Abramo credette a Dio, e gli è stato accreditato come giustizia (vedi anche $\mathrm{Rm}$ 4,397; Gal 3,6 ${ }^{98}$ - nota W.D.). Eb 11,6: Chi si accosta a Dio deve credere,

${ }^{96}$ In Gal., c. 5, lect. 6, n. 332. Pr 3,34: BG: Agli umili concede la sua benevolenza; BT: la sua grazia.

${ }^{97}$ In Rom., c. 4, lect. 1, n. 327: L'Apostolo, dunque, dice: Dico che Abramo così è stato giustificato, che ha la gloria presso Dio (vedi Rm 4,2; In Rom., n. 324 - nota W.D.). Che cosa dice la Scrittura? Gen 15,6: Abramo credette a Dio, il quale gli promise la moltiplicazione della sua discendenza. Sir 2,6: Credi a Dio ed egli ti recupererà. $\mathbf{E}$ gli fu accreditato, cioè da Dio, come giustizia. $1 \mathrm{Mac}$ 2,52: Abramo nella prova fu trovato fedele. E così risulta che /Abramo/ ha la gloria presso Dio, dal quale gli è stato accreditato come giustizia il fatto che credette. Bisogna, poi, considerare che la giustizia, che Dio accredita come scritta, /Abramo/ l'espresse non in qualche opera esteriore, ma nella fede interiore del cuore. Poiché diciamo che c'è un triplice atto di fede, cioè credere Dio, credere a Dio e credere in Dio (credere Deum, Deo, et in Deum), l'Apostolo qui ha posto quell'atto che è credere a Dio (credere Deo), che è il proprio atto di fede, il quale dimostra la sua specificità (specie). Credere in Dio, infatti, dimostra l'ordine della fede al fine, il che avviene per mezzo della carità; credere in Dio, infatti, è, credendo, andare verso Dio (in Deum ire), il che fa la carità. E così è conseguenza della fede specifica (sequitur speciem fidei). Credere che Dio esiste (credere Deum), invece, dimostra la materia della fede, secondo che è virtù teologale (theologica), avendo Dio per oggetto. E perciò questo atto non attinge ancora alla fede specifica (ad speciem fidei), perché se uno, grazie ad alcune ragioni umane e segni naturali, crede che Dio esiste, non diciamo ancora che ha la fede di cui parliamo, ma /lo diciamo/ solo quando crede perché Dio l'ha detto (ex hac ratione credit quod est a Deo dictum), il che è disegnato da ciò che diciamo credere a Dio; e da questo è specificata la fede, così come anche qualsiasi abito conoscitivo ha la sua specie dalla ragione per cui dà l'assenso a qualcosa. Un'altra ragione, infatti, inclina all'assenso proprio della scienza, cioè la dimostrazione, ed un'altra ragione /inclina all'assenso proprio/ dell'opinione, cioè il sillogismo dialettico. Vedi anche S.Th., II-II, q. 2, a. 2 (Utrum convenienter distinguatur actus fidei per hoc quod est credere Deo, credere Deum et credere in Deum; resp. - sì); In III Sent., d. 23, q. 2, a. 2, qc. 2; De Veritate, q. 14, a. 7, ad 7; Super Ioann., c. 6, lect. 3, n. 903. Credere Deum, credere Deo, credere in Deum: vedi S. Agostino, Serm. ad popul., serm. 144, c. 2 (PL 38, 788s); Super Ioann., tr. 29, n. 6 (PL 35, 1631); tr. 59, n. 9s (PL 35, 1778); Enarr. in Psalm., ps. 77 e 78 (PL 36, 988ss), ps. 130 (PL 37, 1704) (nota - W.D.). Sir 2,6: Vlg: Crede Deo et recuperabit te; BG: Affidati a lui ed egli ti aiuterà; BT: Sii fedele a lui ed egli si occuperà di te.

${ }^{98}$ In Gal., c. 3, lect. 3, n. 130: L'Apostolo, dunque, dice: Veramente la giustizia e lo Spirito Santo è dalla fede, come è scritto, Gen 15,6 ed introdotto in $\mathrm{Rm} \mathrm{4,3,} \mathrm{che} \mathrm{Abramo} \mathrm{credette} \mathrm{a} \mathrm{Dio,}$ ecc. (... /segue il discorso sulla giustizia - nota W.D./) E perciò l'Apostolo dice: Abramo credette a Dio, cioè per mezzo della fede sottopose la sua mente a Dio. Sir 2,6: Credi a Dio ed egli ti recupererà, ecc., e più avanti (v. 8 - nota W.D.): Voi, che temete il Signore, credete a Lui, ecc. E gli fu accreditato come giustizia, cioè il credere stesso e la fede stessa fu a lui, ed è a tutti gli altri, la causa sufficiente della giustizia, e che gli è accreditato esteriormente dagli uomini come giustizia, ma interiormente è dato da Dio, il quale coloro che hanno la fede li giustifica mediante la carità operante, perdonando (remittendo) loro i peccati. Sir 2,6.8: Vlg: Crede Deo et recuperabit te, (...) 
ecc $\left({ }^{99}\right)$. E perciò è detto in Sir 1,34: Una cosa beneplacita a Dio è la fede e la mansuetudine, ecc ${ }^{100}$.

D.S. Dockery parla della fedeltà-pistis, "che può significare sia fede che fedeltà. Il contesto, che elenca otto qualità etiche, indica che ci si aspetterebbe una connotazione altrettanto etica della pistis. Poiché Dio è fedele $(\mathrm{Rm} 3,3)$ anche il suo popolo deve essere fedele. Il termine indica l'idea di affabilità e di credibilità" 101 .

Quanto a ciò che è sotto di noi, cioè quanto al corpo - il nostro teologo-esegeta esamina il terzo aspetto - lo Spirito dirige duplicemente:

In primo luogo, quanto agli atti esteriori del corpo, il che avviene mediante la modestia, la quale agli atti stessi oppure ai detti impone un /debito/ modo, e quanto a ciò l'Apostolo dice: modestia. Fil 4,5: La vostra modestia, ecc $\left({ }^{102}\right)$. In secondo luogo, invece, quanto all'appetito sensitivo interiore, e quanto a ciò dice: continenza, la quale fa astenersi anche dalle cose lecite, e castità, la quale fa usare rettamente le cose lecite, come dice la Glossa. Oppure, in un altro modo, continenza si dice dal fatto che l'uomo può essere assalito dalle cattive concupiscenze, tuttavia tiene se stesso mediante il vigore della ragione e non si lascia portar via; e perciò il nome di continenza viene assunto dal fatto che uno tiene se stesso contro l'assalto. Castità invece

Qui timetis Dominum, credite illi; BG: Affidati a lui ed egli ti aiuterà, (...) confidate in lui; BT: Sii fedele a lui ed egli si occuperà di te, (...) credete a lui.

${ }^{99}$ In Hebr., c. 11, lect. 2, n. 575: Chi si accosta a Dio deve credere. Nessuno infatti può piacere a Dio se non si accosta a Lui. Gc 4,8: Avvicinatevi a Dio ed Egli si avvicinerà a voi. Sal 34 133/,5: Accostatevi a Lui e sarete illuminati. Ma nessuno si accosta a Dio se non per mezzo della fede, perché la fede è il lume dell'intelletto. Dunque nessuno può piacere a Dio se non per mezzo della fede. Bisogna però che chi si accosta per mezzo della fede creda al Signore. Sal 34/33/,5: Vlg: Accedite ad eum, et illuminamini; BG, Sal 34/33/,6: Guardate a lui e sarete raggianti; BT: Guardate a lui, raggiate di gioia.

${ }^{100}$ In Gal., c. 5, lect. 6, n. 333; vedi anche S.Th., II-II, q. 4, a. 5 (Utrum fides sit virtus; resp. - sì), ad 4 (dove c'è la risposata all'obiezione presa da Gal 5,23); q. 8, a. 8 (Utrum in fructibus fides respondeat dono intellectus; resp. - sì); In III Sent., d. 34, q. 1, a. 5. La fede è una cognizione certa delle cose invisibili: vedi Glossa interl., super Gal. 5,23 (VI, 87v); Pietro Lombardo, Glossa super Gal. 5,23 (PL 192, 160) (nota - W.D.). Sir 1,34: BG e BT, Sir 1,27/34/: Egli si compiace della fedeltà e della mansuetudine.

${ }^{101}$ D.S. Dockery, Frutto dello Spirito, op. cit., p. 655.

${ }^{102}$ In Philipp., c. 4, lect. 1, n. 153 (Modestia vestra nota sit omnibus hominibus; BG: La vostra amabilità; BT: La vostra indulgente mitezza): /Il gaudio/ deve essere anche moderato, cioè non scorra fuori mediante la voluttà, come lo fa il gaudio del mondo. E perciò l'Apostolo dice: la vostra modestia, ecc.; come se dicesse: Il vostro gaudio sia così moderato che non si cambi nella dissolutezza. Gdt 16,24: Il popolo era giocondo secondo la faccia dei santi, ecc. Pr 12,11: Chi è soave vive nelle moderazioni. E dice: sia nota a tutti gli uomini; come se dicesse: La vostra vita sia così moderata nelle cose esteriori che nessuno ne offenda l'aspetto: l'impedirà infatti il vostro comportamento. Gdt 16,24: Vlg: Populus erat iucundus secundum faciem sanctorum; BG, Gdt 16,20: Il popolo continuò far festa a Gerusalemme vicino al tempio; BT, Gdt 16,20: Il popolo si rallegrava davanti al tempio a Gerusalemme. - Pr 12,11: BG e BT: Chi coltiva la sua terra si sazia di pane. 
si dice dal fatto che uno né viene assalito, né portato via, e si dice dal castigo ( $a$ castigando). Diciamo infatti che è ben castigato chi si tiene ordinatamente in tutte le $\operatorname{cose}^{103}$.

D.S. Dockery invece parla della mitezza e del dominio di sé. La mitezza, secondo lui, "combina la fermezza e l'umiltà, denotando fermezza sotto controllo. Essa manca di ogni senso negativo, di assenza di spirito, coraggio o vigore, a volte associato con il termine mitezza. Questa grazia spirituale è meglio comprensibile come umile disposizione verso la volontà divina (Burton 317 ${ }^{104}$ ). Dominio di sé - Quest'ultimo termine è particolarmente importante. L'idea è stata introdotta nell'etica greca da Socrate e, al tempo di Paolo, il termine è diventato un concetto primario nel pensiero ellenistico. La sua collocazione alla fine della lista indica, probabilmente, che proprio come l'amore, la prima qualità, rappresenta l'adempimento della Legge, il dominio di sé adempie la richiesta completa dell'etica greca $^{105 "}$ ".

San Tommaso però non si ferma, ma, volendo completare la lezione, risolve ancora due dubbi $\left({ }^{106}\right)$. Il primo riguarda il fatto che i frutti dello Spirito si oppongono alle opere della carne, perciò sembra che l'Apostolo avrebbe dovuto elencare tanti frutti dello Spirito, quante opere della carne aveva elencato in Gal 5,19-21, ma non l'ha fatto. Non l'ha fatto - risponde brevemente l'Aquinate - perché i vizi sono più numerosi delle virtù. Il secondo dubbio invece riguarda il fatto che i frutti dello Spirito qui elencati non corrispondono alle opere della carne. L'Apostolo - risponde l'Angelico - non intende trasmettere qui una dottrina sistematica sui vizi e sulle virtù, e perciò non pone un vizio contro una virtù, ma enumera alcuni dei vizi ed alcune delle virtù, secondo lo svolgersi dell'intenzione della presente lettera. Tuttavia però - osserva il nostro teologo-esegeta - se consideriamo diligentemente, vediamo che in qualche modo corrispondo a sé contrariamente. Infatti, contro la fornicazione, che è l'amore illecito, corrisponde la carità; contro l'immondizia, l'impudicizia e la lussuria, che sono incitamenti carnali e provengono dalla fornicazione, viene posto il gaudio che è godimento spirituale conseguente dalla carità; contro l'idolatria viene posta la pace; contro gli incantesimi ecc. fino ai dissensi vengono poste la pazienza, la longanimità

${ }^{103}$ In Gal., c. 5, lect. 6, n. 334; vedi anche S.Th., II-II, q. 134, a. 1 (Utrum magnificentia sit virtus; resp. - sì); q. 151, a. 1 (Utrum castitas sit virtus; resp. - sì); q. 155, a. 1 (Utrum continentia sit virtus; resp. - sì); q. 160, a. 1 (Utrum modestia sit pars temperantiae; resp. - sì); q. 161 (De humilitate), Prol.; De Veritate, q. 14, a. 4; In Ethic., lib. 3, lect. 22; lib. 4, lect. 11; In Hebr., c. 12, lect. 2, n. 674; In II Sent., d. 44, q. 2, a. 1, ad 3; In III Sent., d. 33, q. 3, a. 2, qc. 1 e ad 1; S.Th., I-II, q. 58, a. 3, ad 2; III, q. 7, a. 2, ad 3. Glossa: Pietro Lombardo, Glossae, col. 160 (PL 192 compl. W.D.); cfr. Glossa interl. (VI, 77v) (nota - W.D.). Castità si dice a castigando: vedi Aristotele, Etica, lib. 3, 12, 5 (1119 a 33) (nota - W.D.).

${ }^{104}$ E.D. Burton, The Epistle to the Galatians, ICC, T. e T. Clark, Edinburgh 1921, p. 317.

${ }^{105}$ D.S. Dockery, Frutto dello Spirito, op. cit., pp. 655-656.

${ }^{106}$ Cfr. D.S. Dockery, Frutto dello Spirito, op. cit., p. 654. 
e la bontà; contro le sette la fede; contro gli omicidi la mansuetudine; contro l'ubriachezza, la gola e ad esse simili, vengono poste la modestia, la continenza e la castità $^{107}$.

\section{Contro queste cose non c’è Legge - Gal 5,23b}

Nella nota a questo testo, la BG dice: "Legge: il credente unito a Cristo non ha più una Legge che gli detti la sua condotta dall'esterno. Egli compie la legge dello Spirito (vv. 18.23.25; 6,2; Rm 6,15; 8,2-4; Fil 1,9-10; cfr. Gc 1,25; 2,8)" "108.

U. Vanni, commentando Gal 5,23b, dice: "La Legge giudaica non ha a che fare, non si occupa, non ha un rapporto moralmente valido con cose del genere, che dipendono esclusivamente dallo Spirito" ${ }^{109}$.

San Tommaso invece pone l'accento sulla liberazione dalla Legge, benché con altre parole e riferimenti biblici, ma non indicati:

Dopo aver enumerato le opere della carne e quelle dello Spirito, l'Apostolo conclude conseguentemente da entrambe le parti: che coloro che seguono lo Spirito non sono sotto la Legge. Ed usa una tale prova: È sotto la Legge chi è soggetto alla Legge (obnoxius legi $\left.{ }^{110}\right)$, cioè chi fa le cose contrarie alla Legge; ma quelli che agiscono /mossi/ dallo Spirito (cfr. Rm 8,14 ${ }^{111}$ - nota W.D.), non fanno le cose contrarie alla Legge,

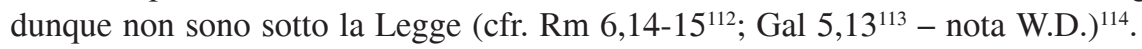

${ }^{107}$ Vedi In Gal., c. 5, lect. 6, n. 335; vedi anche S.Th., I-II, q. 70, a. 1 (Utrum fructus Spiritus Sancti quos Apostolus nominat ad Galatas 5, sint actus; resp. - sì); a. 3 (Utrum fructus convenienter enumerentur ab Apostolo; resp. - sì), ad 3 (e non ad 4, come ha l'Ed. Marietti 1953, vol. 1, p. 637); a. 4 (Utrum fructus Spiritus Sancti contrarientur operibus carnis; resp. - sì).

${ }^{108}$ BG, ed. cit., p. 2779.

${ }^{109}$ U. Vanni, Lettera ai Galati, in P. Rossano (a cura di), Lettere di san Paolo, op. cit., p. 257.

${ }^{110}$ Cfr. Eb 2,15; In Hebr., c. 2, lect. 4, n. 145 (Eb 2,15: e liberare così quelli che, per timore della morte, erano soggetti a schiavitù per tutta la vita - et liberaret eos qui timore mortis per totam vitam obnoxii erant servituti): Oppure in un altro modo (cfr. ibid., n. 144 - nota W.D.): L'uomo infatti era soggetto (obnoxius) ad una duplice schiavitù, cioè della Legge e del peccato. Perciò, in At 15,10, la Legge viene detta giogo che né i nostri padri né noi siamo in grado di portare. Le mani di Mosè infatti erano pesanti, Es 17,12; Gal 4,5: Per redimere coloro che erano sotto la Legge. Erano infatti soggetti (obnoxii) alla schiavitù del peccato. Da questa duplice schiavitù Cristo ci ha liberati. La differenza, poi, tra il Nuovo e il Vecchio Testamento è /come quella tra/ il timore e l'amore. Nel Nuovo c'è l'amore. Gv 14,15: Se mi amate, osservate i miei comandamenti. Il Vecchio/Testamento/ invece fu legge del timore. $\mathrm{Rm}$ 8,15: Non avete ricevuto uno spirito della schiavitù per ricadere nel timore. E perciò è detto: Per liberare quelli che, per timore della morte corporale che la Legge infliggeva, per tutta la vita erano soggetti alla schiavitù della Legge.

${ }^{111}$ In Rm., c. 8, lect. 3, n. 635 (Rm 8,14: Infatti tutti quelli che sono guidati dallo Spirito di Dio, questi sono figli di Dio - Quicumque enim spiritu Dei aguntur, ii sunt filii Dei): Qui, in primo luogo, bisogna considerare in che modo alcuni agiscono /mossi/ dallo Spirito di Dio. E si può così intendere: Quelli che agiscono /mossi/ dallo Spirito di Dio, cioè vengono guidati (reguntur) come da un conduttore e direttore, il che appunto in noi fa lo Spirito, cioè in quanto ci illumina interiormente circa ciò che dobbiamo fare. Sal 143/142/,10: Il tuo Spirito buono mi conduca, ecc. Ma per- 


\section{E continua:}

L'Apostolo dunque dice: Dico che quelli che agiscono /mossi/ dallo Spirito, non fanno le opere contrarie alla Legge, perché fanno le opere dello Spirito, e contro queste cose non c'è Legge, cioè contro le opere dello Spirito, ma lo Spirito le insegna. Come

ché quello che è condotto non opera da se stesso, l'uomo spirituale invece non solo è istruito dallo Spirito Santo circa ciò che deve fare, ma anche il suo cuore è mosso dallo Spirito Santo, perciò in queste parole bisogna intendere più di ciò che è detto: quelli che agiscono /mossi/ dallo Spirito di Dio. Diciamo che agiscono /mossi/ quelli che vengono mossi da uno stimolo superiore. Perciò degli animali diciamo che non agiscono, ma vengono spinti ad agire (sed aguntur), perché vengono mossi a compiere le loro azioni dalla natura e non dal proprio moto. Similmente poi l'uomo spirituale: non quasi dal moto della propria volontà principalmente, ma dallo stimolo dello Spirito Santo viene inclinato ad agire qualcosa, secondo ciò che in Is 59,19: Verrà come un fiume violento, sospinto dallo Spirito di Dio; e in Lc 4,1 /è detto/ che Cristo era guidato (agebatur) dallo Spirito nel deserto. Per questo però non si esclude che gli uomini spirituali operino per mezzo della volontà e del libero arbitrio, perché il moto stesso della volontà e del libero arbitrio, causa in essi lo Spirito Santo, secondo ciò che in Fil 2,13: È Dio infatti che suscita in voi il volere e l'operare. Vedi anche S.Th., I-II, q. 93, a. 6, ad 1; q. 68, aa. 1ss; II-II, q. 52, a. 1, ad 3. Is 59,19: BG: dal vento del Signore; BT: dal soffio del Signore; Vlg: quem spiritus Domini cogit.

${ }^{112}$ In Rom., c. 6, lect. 3, nn. 497-501 (Rm 6,14-15: /ll peccato infatti non dominerà su di voi/, perché non siete sotto la Legge, ma sotto la grazia. Che dunque? Ci metteremo a peccare perché non siamo sotto la Legge, ma sotto la grazia? È assurdo! - Non enim sub lege estis, sed sub gratia. Quid ergo? Peccabimus, quoniam non sumus sub lege, sed sub gratia? Absit.): Qui l'Apostolo dimostra ciò che ha detto, dicendo: Non siete sotto la Legge, ma sotto la grazia. Qui bisogna considerare che qui non si parla della Legge soltanto quanto ai /precetti/ cerimoniali, ma anche quanto ai /precetti/ morali. E in questo modo anche Cristo fu sotto la Legge, secondo ciò che in Gal 4,4: Nato sotto la Legge, perché cioè aveva osservato la Legge non soltanto quanto ai /precetti/ morali, ma anche quanto ai /precetti/ cerimoniali. I fedeli di Cristo, poi, in un certo modo sono sotto la Legge, quanto ai /precetti/ morali, ma non quanto ai /precetti/ cerimoniali. In un altro modo si dice che uno è sotto la Legge, quasi forzato (coactus) dalla Legge; e così si dice che è sotto la Legge chi non volontariamente dall'amore, ma dalla paura è costretto ad osservare la Legge. In un tale però manca la grazia, la quale, se vi fosse, inclinerebbe la volontà all'osservanza della Legge, affinché dall'amore compisse i /precetti/ morali di essa. Così dunque, finché uno in questo modo è sotto la Legge, che non la compie volontariamente, in lui domina il peccato che inclina la volontà dell'uomo affinché voglia ciò che è contrario alla Legge, ma per mezzo della grazia un tale dominio viene tolto, affinché cioè l'uomo osservi la Legge, non quasi esistendo sotto la Legge, ma come libero. Gal 4,31: Non siamo figli della schiava, ma della libera: Cristo ci ha liberati per questa libertà. Questa grazia, poi, che fa sì che l'uomo liberamente compie la Legge, non la conferivano i sacramenti della Legge (legalia), ma la conferiscono i sacramenti di Cristo; e perciò quelli che si sottomettevano ai /precetti/ cerimoniali della Legge - per quanto riguarda la forza dei sacramenti della Legge - non erano sotto la grazia, ma sotto la Legge, a meno che non abbiano ricevuto la grazia per mezzo della fede di Cristo. Quelli invece che si sottomettono ai sacramenti di Cristo, dalla loro forza ricevono la grazia, affinché non siano sotto la Legge, ma sotto la grazia, a meno che - forse per colpa loro - non si sottomettano alla schiavitù del peccato. Quando l'Apostolo dice: Che dunque?, ecc., pone una questione contro ciò che ha detto. (...) Bisogna dunque considerare che alcuni potrebbero intendere male le suddette parole dell'Apostolo riguardanti alcune cose, che cioè i fedeli di Cristo non siano sotto la Legge quanto all'obbligo dell'osservanza dei precetti morali, da che risulterebbe che ai fedeli di Cristo sia permesso (licitum) di peccare, cioè comportarsi contro i precetti morali. E perciò, sotto questo senso, l'Apostolo pone la questione, dicendo: Che 
infatti la Legge insegna esteriormente le opere delle virtù, così anche lo Spirito muove interiormente ad esse. Rm 7,22: Infatti nel mio intimo acconsento alla legge di Dio, ecc. Oppure, fanno le opere della carne, e queste in quelli che agiscono /mossi/ dallo Spirito di Dio, non sono contrarie alla Legge ${ }^{115}$.

Se lasciassimo qui l'esposizione dell'Aquinate, impoveriremmo il suo pensiero, perché egli commenta il v. 23b assieme al v. 24: Quelli che sono di Cristo Gesù hanno crocifisso la carne con le sue passioni e i suoi desideri - Qui autem sunt Christi, carnem suam crucifixerunt cum vitiis et concupiscentiis.

Commentando questo versetto, U. Vanni dice che "in forza della relazione intima con Cristo determinatasi col battesimo, i cristiani hanno crocifisso la loro debolezza umana, la carne, con tutti i suoi germi negativi, passioni e desideri sfrenati, in quanto l'hanno trasferita in Cristo crocifisso, appropriandosi la sua vita divina, il suo Spirito" 116 .

Anche l'Angelico, a modo suo, parla delle stesse cose, con consigli spirituali molto attuali, e dice così:

Quelli invece che sono di Cristo, cioè quelli che hanno lo Spirito di Dio. Rm 8,9:

Chi non ha lo Spirito di Dio, non è di Lui $\left.{ }^{117}\right)$. Quelli dunque agiscono /mossi/ dallo

dunque diremo, che peccheremo, cioè comportandoci contro i precetti morali, perché è detto che non siamo sotto la Legge, ma sotto la grazia? E questo senso l'Apostolo lo disapprova in Gal 5,13: Voi, fratelli miei, siete stati chiamati a libertà: purché questa libertà non divenga un'occasione di darvi alla carne. E perciò, rispondendo, subito aggiunge: Non ci sia, che cioè pecchiamo perché siamo liberati dalla Legge, poiché se peccheremo, ciò risulterà inconveniente, perché di nuovo ritorneremmo nella schiavitù del peccato. Vedi anche S.Th., I-II, q. 103, a. 2 (Utrum caeremoniae veteris legis habuerint virtutem iustificandi tempore legis; resp. - no); q. 100, a. 12; q. 102, a. 5, ad 4; III, q. 62, a. 6 (Utrum sacramenta veteris legis gratiam causarent; resp. - no); q. 72, a. 5, ad 3; In IV Sent., d. 1, q. 1, a. 5, qcc. 1 e 3; d. 18, q. 1, a. 3, qc. 1, ad 1; De Veritate, q. 27, a. 3, ad 20; q. 28, a. 2, ad 12; C.G., IV, c. 57; In Gal., c. 2, lect. 4; nn. 94-96; c. 3, lect. 4, n. 136; In Hebr., c. 9, lect. 3, n. 443. Gal 4,31: così la Vlg; BG e BT: Gal 4,31 - 5,1 (nota - W.D.).

${ }^{113}$ In Gal., c. 5, lect. 3, nn. 299-300 (Gal 5,13): La condizione di ogni stato riguarda la schiavitù o la libertà. Ma lo stato della fede di Cristo, al quale conduce l'Apostolo, riguarda la libertà ed è la libertà stessa. E perciò dice: Voi, fratelli (...) siete stati chiamati, cioè da Dio, alla libertà della grazia. $\mathrm{Rm}$ 8,15: Non avete ricevuto uno spirito della schiavitù, per ricadere nella paura, ma avete ricevuto lo spirito dell'adozione a figli. Gal 4,31: Non siamo figli della schiava, ma della libera, ecc. (...) C'è però l'abuso di /questo/ stato: se esso viene trascinato indietro e la libertà dello spirito viene cambiata in schiavitù della carne. I Galati erano già liberi dalla Legge, ma perché non credessero che fosse loro permesso di commettere i peccati che la Legge proibiva, l'Apostolo indica l'abuso della libertà, dicendo: purché questa libertà non ecc., come se volesse dire: Siete liberi, così però che non abusiate della vostra libertà, pensando che vi sia permesso di peccare impunemente. 1 Cor 8,9: Badate che la vostra libertà (licentia) non divenga occasione di caduta per i deboli. Gal 4,31: così la Vlg; BG e BT: Gal 4,31 - 5,1 (nota - W.D.).

${ }^{114}$ In Gal., c. 5, lect. 7, n. 336.

${ }^{115}$ In Gal., c. 5, lect. 7, n. 337.

${ }^{116}$ U. Vanni, Lettera ai Galati, in P. Rossano (a cura di), Lettere di san Paolo, op. cit., p. 258.

${ }^{117}$ Qui san Tommaso ha cambiato il testo di Rm 8,9; In Rom., c. 8, lect. 2, n. 627 (Se qualcuno 
Spirito di Dio, questi sono di Cristo. Questi, dico, hanno crocifisso la loro carne, ecc. Non dice però: evitano i vizi e le concupiscenze, perché un buon medico allora cura bene, quando applica i rimedi contro la causa del morbo. La carne invece è la radice dei vizi. Se dunque vogliamo evitare i vizi, dobbiamo domare la carne. 1 Cor 9,27: Castigo il mio corpo, ecc. Poiché però la carne viene domata mediante le veglie, i digiuni e i lavori - Sir 33,28: Al servo malevolo tortura e ceppi, ecc (mettilo al lavoro perché non si liberi - W.D. con Vlg). - a queste opere invece /i fedeli/ vengono mossi dalla devozione che hanno verso il Cristo crocifisso, perciò l'Apostolo dice significatamene: hanno crocifisso, cioè si sono conformati al Cristo crocifisso, affliggendo la loro carne, ecc. Rm 6,6: Il nostro uomo vecchio è stato crocifisso insieme /con lui/, ecc( $\left.{ }^{118}\right)$. Gal 2,19: Affinché io viva per Dio; sono stato crocifisso, ecc

non ha lo Spirito di Cristo, non gli appartiene - Si quis autem Spiritum Christi non habet, hic non est eius): Qui l'Apostolo fa vedere che questa condizione (si tratta di Rm 8,9 secondo la Vlg: se lo Spirito di Dio abita in voi; BG: dal momento che lo Spirito di Dio abita in voi; BT come Vlg; vedi In Rom., n. 626 - nota W.D.) deve essere compiuta. Dice: se qualcuno non ha lo Spirito di Cristo, questi non è di Lui. Come non è membro del corpo ciò che non è vivificato dallo spirito del corpo, così non è membro di Cristo chi non ha lo Spirito di Cristo. $1 \mathrm{Gv} 4,13$ : In questo si conosce che noi rimaniamo in lui ed egli in noi: egli ci ha donato il suo Spirito. Bisogna però notare che lo stesso è lo Spirito di Cristo e /lo Spirito/ di Dio Padre; ma si dice di Dio Padre in quanto procede dal Padre; si dice Spirito di Cristo in quanto procede dal Figlio. Perciò anche il Signore sempre l'attribuì simultaneamente a se e al Padre, come in Gv 14,26: Il Paraclito, lo Spirito Santo che il Padre manderà nel mio nome. Anche (Gv 15,26 - nota W.D.): Quando verrà il Paraclito, che io vi manderò dal Padre, ecc.

${ }^{118}$ In Rom., c. 6, lect. 2, nn. 479-481 (Rm 6,6: Lo sappiamo: l'uomo vecchio che è in noi è stato crocifisso con lui, affinché fosse reso inefficace questo corpo di peccato, e noi non fossimo più schiavi del peccato - Hoc scientes quia vetus homo noster simul crucifixus est, ut destruatur corpus peccati, et ultra non serviamus peccato): L'Apostolo dunque dice: È stato detto che dobbiamo camminare nella novità, cioè desistere dal peccato. E perché qualcuno non dica che questo è impossibile, dice: lo sappiamo che il nostro uomo vecchio, cioè la vecchiezza dell'uomo introdotta dal peccato, insieme, cioè con Cristo, è stato crocifisso, cioè è stato mortificato per mezzo della croce di Cristo. Come abbiamo sopra detto (vedi In Rom., n. 476 - nota W.D.), la vecchiezza dell'uomo è stata introdotta per mezzo del peccato, in quanto per mezzo del peccato si corrompe la bontà della natura; questa vecchiezza appunto domina (principatur) nell'uomo finché l'uomo soggiace al peccato. E poiché ciò che nell'uomo è principale lo diciamo uomo stesso, perciò in colui che soggiace al peccato la vecchiezza stessa del peccato si dice uomo vecchio. La vecchiezza del peccato poi può essere intesa o come reato stesso, cioè la macchia dei peccati attuali, o anche come consuetudine di peccare che introduce una certa necessità di peccare, o anche il fomento del peccato proveniente dal peccato dei primi genitori. Così dunque diciamo che il nostro uomo vecchio è stato crocifisso in insieme con Cristo, in quanto la predetta vecchiezza è stata rimossa per virtù di Cristo. O perché è stata totalmente rimossa, così come nel battessimo viene totalmente rimosso il reato e la macchia del peccato. O perché viene diminuita la sua forza, come la forza del fomento, o anche la consuetudine di peccare. Col 2,14: Ha annullato il documento scritto contro di noi che, con le prescrizioni, ci era contrario: lo ha tolto di mezzo inchiodandolo alla croce. In seguito, l'Apostolo pone il duplice effetto del predetto beneficio. Il primo di essi è la rimozione dei delitti precedenti. E ne dicono le parole: perché fosse distrutto il corpo del peccato. Corpo del peccato si dice infatti l'insieme delle opere cattive, così come l'insieme dei membri fa un solo corpo naturale. Gb 41,7/6/: Il suo corpo come scudi fusi, ecc (e compatto con le squame prementi se stesse - W.D. con Vlg). Il secondo effetto invece è che ci guardiamo dai peccati in futuro: e ne dicono le parole 
(con Cristo $^{119}$ ). Che poi non crocifiggono la carne distruggendo la natura, perché nessuno ha in odio la propria carne, come è detto in Ef 5,29(120), ma quanto alle cose che sono contrarie alla Legge, perciò l'Apostolo dice: con i vizi, cioè con i peccati, e le concupiscenze, cioè le passioni, dalle quali l'anima viene inclinata a peccare. Infatti, non crocifigge bene la carne chi lascia anche lo spazio alle passioni, altrimenti - siccome la ragione non sempre vigila come si deve per evitare i peccati - talvolta può cadere. Sir 18,30: Non andare dietro le tue concupiscenze, ecc. Rm 13,14( $\left.{ }^{121}\right)$ : Non abbiate cura della carne nei suoi desideri ${ }^{122}$.

E, per concludere, vediamo Gal 5,25: Perciò se viviamo dello Spirito, camminiamo anche secondo lo Spirito, perché, come osserva D.S. Dockery, "Gal 5,25 fornisce un versetto sintetico per questa sezione, mediante la ripetizione del dati-

soggiunte: e non servissimo più il peccato. Allora infatti l'uomo serve il peccato, quando obbedisce alla concupiscenza del peccato per mezzo del consenso e l'esecuzione corporale. Gv 8,34: Chi commette il peccato è schiavo del peccato. Vedi anche S.Th., III, q. 69, a. 3, sed contra; Pietro Lombardo, Glossa super Rom. 6,6 (PL 191, 1404; S. Agostino, De Pecc. Remiss. et Bapt. Parv., lib. 1, c. 39 (PL 44, 150). Gb 41,7/6/: BG: Il suo dorso è formato da file di squame, saldate con tenace suggello; BT: Il suo dorso le file di squame, compatto come chiuso con un sigillo.

${ }^{119}$ In Gal., c. 2, lect. 6, n. 106: Qualsiasi uomo, secondo l'origine della natura, nasce come figlio dell'ira, Ef 2,3: Eravamo infatti per natura figli dell'ira, ecc. Nasce anche nella vecchiezza del peccato, Bar 3,10: Invecchiasti nella terra straniera, ecc. Questa vecchiezza appunto viene tolta per mezzo della croce di Cristo, e conferisce la novità della vita spirituale. L'Apostolo dunque dice: Sono stato crocifisso con Cristo, cioè la concupiscenza, ossia il fomento del peccato, ed ogni cosa del genere è morta in me per mezzo della croce di Cristo. Rm 6,6: Il nostro uomo vecchio è stato crocifisso insieme, ecc.

${ }^{120}$ In Eph., c. 5, lect. 9, n. 327: Che poi così bisogna amare se stesso (si tratta di Ef 5,28: chi ama la propria moglie, ama se stesso; vedi In Eph., n. 326 - nota W.D.), l'Apostolo lo dimostra dicendo: nessuno infatti ha in odio la propria carne; il che risulta mediante l'effetto, perché "la prova dell'amore è la prestazione delle opere". Infatti, amiamo ciò che conserviamo per le energie (pro viribus). Ma ciascuno nutre e cura la propria carne per la conservazione. $1 \mathrm{Tm}$ 6,8: Quando dunque abbiamo di che mangiare e di che coprirci, ecc. "la prova ... delle opere": probatio dilectionis est exhibitio operis: S. Gregorio Magno, In Evang., lib. 2, hom. 30 (PL 76, 1220) (nota W.D.).

${ }^{121}$ In Rom., c. 13, lect. 3, n. 1080 (Rm 13,14: Non lasciatevi prendere dai desideri della carne - Et carnis curam ne feceritis in desideriis): Qui l'Apostolo spiega in che modo dobbiamo comportarci onestamente, come in pieno giorno (Rm 13,13; vedi In Rom., n. 1073 - nota W.D.), perciò soggiunge: non abbiate cura della carne nei suoi desideri. La bellezza dell'onestà infatti consiste in questo: che l'uomo non preferisca allo spirito la carne, ma alla carne lo spirito. Rm 8,12: Siamo debitori non della carne per vivere secondo la carne. Ma bisogna notare che l'Apostolo non dice semplicemente: non abbiate cura della carne, perché ciascuno è tenuto di avere cura della carne, perché esso, per sua natura, esige il sostentamento, secondo ciò che in Ef 5,29: Nessuno infatti ha in odio la propria carne, ma la nutre e la cura, ecc. Ma aggiunge: nei suoi desideri, affinché non seguiamo i disordinati desideri della carne, cioè le concupiscenze. Perciò è detto in Gal 5,16: Camminate secondalo Spirito e non sarete portati a soddisfare i desideri della carne.

${ }^{122}$ In Gal., c. 5, lect. 7, n. 338. Sir 33,28: Vlg: Servo malivolo tortura et compedes, mitte illum in operatione ne vacet; BG e BT, Sir 33,27-28: Per lo schiavo malvagio torture e castighi. Mettilo a lavorare perché non sita in ozio; BG nella nota: "perché non stia in ozio: l'ebr. ha: perché non si rivolti”. - Sir 18,30: BG e BT: Non seguire le passioni, poni un freno ai tuoi desideri. 
vo «Spirito» (pneumati). L'enfatico sommario di Paolo è più comprensibile come esortazione a «comportarsi » (stoikōmen) secondo lo Spirito. Il contesto sottolinea la potenza dello Spirito come motivazione e capacità del vivere cristiano. La concezione paolina della vita nello Spirito viene formulata sia in modo positivo che negativo. Le virtù (il frutto dello Spirito) sono esempi genuini del carattere etico prodotto in coloro che camminano secondo lo Spirito" 123.

San Tommaso, nell'interpretazione di Gal 5,25, dà una spiegazione e un'esortazione spirituale:

Dobbiamo camminare per mezzo dello Spirito, perché anche viviamo grazie a Lui, e non grazie alla carne. $\mathrm{Rm} 8$,12: Siamo debitori non della carne, ecc $\left({ }^{124}\right)$. Se dunque viviamo dello Spirito, dobbiamo in tutto agire /mossi/ da Lui (ab ipso agi). Infatti, come nella vita corporale il corpo non si muove se non mediante l'anima, grazie alla quale vive, così nella vita spirituale ogni moto deve essere dallo Spirito Santo. Gv 6,63/64/: È lo Spirito che dà la vita $\left({ }^{125}\right)$. At 18,28: In lui viviamo, ci muoviamo e siamo $^{126}$.

\section{Conclusione}

Nella Super Epistolas S. Pauli Lectura, che san Tommaso ha pensato ed effettuato come opera cristologica e cristocentrica ${ }^{127}$, troviamo anche una, molto ricca, dottrina sui frutti dello Spirito Santo, cioè sulle virtù indicate da questi frut-

${ }^{123}$ D.S. Dockery, Frutto dello Spirito, op. cit., p. 653.

${ }^{124}$ In Rom., c. 8, lect. 2, nn. 632-633 (Rm 8,12-13: Ergo, fratres, debitores sumus non carni, ut secundum carnem vivamus, si enim secundum carnem vixeritis, moriemini; si autem spiritu facta carnis mortificaveritis, vivetis): Abbiamo detto che grazie allo Spirito Santo pervengono a noi molti doni e che dalla prudenza della carne viene la morte. Dunque, siamo debitori dello Spirito Santo, a motivo dei benefici da Lui ricevuti, affinché viviamo secondo lo Spirito e non secondo la carne. Gal 5,25: Se viviamo dello Spirito, camminiamo anche secondo lo Spirito. In seguito, l'Apostolo assegna la ragione di questa conclusione. $\mathrm{E}$, in primo luogo, quanto alla carne, dicendo: se infatti vivrete secondo la carne, cioè secondo le concupiscenze della carne, morirete, cioè di

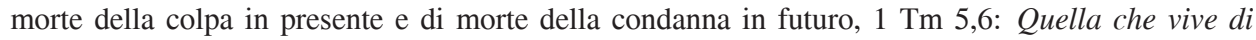
piaceri, è già morta. In secondo luogo, assegna la ragione quanto allo Spirito, dicendo: se invece con lo Spirito, cioè mediante lo Spirito, mortificherete i fatti della carne, cioè le opere che provengono dalla concupiscenza della carne, vivrete, di vita della grazia in presente e di vita della gloria in futuro. Col 3,5: Mortificate i vostri membri che sono sulla terra. Gal 5,24: Quelli che sono di Cristo hanno crocifisso la loro carne con i vizi e le concupiscenze.

${ }^{125}$ Super Ioann., c. 6, lect. 8, n. 998: /Queste parole del Signore/ possono essere intese in due modi, cioè secondo il significato spirituale e secondo il significato corporale. E perciò /il Signore/ dice: $\mathbf{E}$ lo Spirito che dà la vita, cioè: se le parole che vi ho detto voi le intendete spiritualmente, vivrete. La carne non giova a nulla, cioè: se le intendete secondo il loro significato corporale, non vi gioveranno a nulla, ma piuttosto vi danneggeranno. Infatti, è detto in $\mathrm{Rm}$ 8,13: Se vivrete secondo la carne, morirete (trad. - W.D.).

${ }^{126}$ In Gal., c. 5, lect. 7, n. 340.

127 Vedi In Rom., Prologo, nn. 5-11. 
ti, strettamente legata alla dottrina dei vizi contrari a questi frutti, con dei consigli morali e spirituali validi anche oggi.

Dobbiamo notare che nei tempi successivi, fino ai nostri tempi, l'esegesi biblica diventerà sempre più precisa, più scientifica e più tecnica, ma la profondità spirituale non ne guadagnerà molto.

Come abbiamo visto, in questa opera esegetica dell'Aquinate la sua dottrina - esposta sempre in occasione delle parole usate nel testo sacro - non si basa sulla speculazione filosofica, come nelle sue opere sistematiche, ma sulla giusta interpretazione teologica dei testi della Sacra Scrittura ${ }^{128}$. In questa sua opera l'Angelico usa un metodo dell'analisi ed interpretazione dei testi biblici molto efficace, cioè il metodo della lettura e spiegazione della Sacra Scrittura con ed alla luce della stessa Sacra Scrittura. Questo metodo medievale dell'interpretazione dei testi biblici con i testi paralleli arricchisce e completa sia il testo stesso, sia la dottrina che viene esposta, e fa sì che la formulazione della dottrina di san Tommaso sui frutti dello Spirito Santo (e sui vizi ad essi opposti), esposta in questa sua opera esegetica, è una formulazione molto intelligente che costituisce un raro esempio di equilibrata sintesi tra la Bibbia, di cui l'Aquinate si dimostra un ottimo conoscitore, la Tradizione e la speculazione o, meglio dire, riflessione teologica; ma allo stesso tempo è anche una formulazione molto "moderna" ed attuale, sia nel linguaggio che nei concetti: un linguaggio biblico, ricco di citazioni ben scelte, esprime i concetti di questa dottrina e permette di rimanere nell'ambito della fede, della morale e della spiritualità della Chiesa.

\section{Owoce Ducha Świętego - komentarz św. Tomasza z Akwinu do Ga 5,22nn}

\section{Streszczenie}

We Wstępie - aby rozwiać błędne, ale rozpowszechnione mniemania o działalności uniwersyteckiej św. Tomasza z Akwinu - Doktor Anielski, który nigdy nie wykładał swojej Summy teologii, jest przedstawiony jako egezegeta Pisma Świętego, gdyż w jego czasach profesor teoglogii był magister in Sacra Pagina, czyli zajmował się właśnie egzegezą Pisma Świętego. Ponieważ lista owoców Ducha Świętego w Ga 5,22-23 jest porzedzona listą wad, najpierw przedstawiona jest nauka św. Tomasza o wymienionych tam wadach-grzechach, a następnie owoce Ducha Świętego na podstawie Ga 5,22-23, czyli cnoty związane z tymi owocami, wreszcie Ga 5,24-25 o krzyżowaniu swego ciała z jego namiętnościami i pożądaniami oraz o życiu według Ducha. Teksty kometarzy Akwinaty są konfrontowane ze współczesnymi komentarzami biblijnymi, co pozwala na zauważenie różnic w podejściu do tekstów świętych, a także dostrzeżenie bogactwa myśli teologicz-

${ }^{128}$ Vale la pena di notare che in quest'opera di san Tommaso sono menzionati i filosofi: Aristotele - 76 volte, Platone ed i platonici - 13, Avicenna - 2, Anassagora - 1, Empedocle - 1, in generale i Filosofi - 4; vedi Ed. Marietti 1953, vol. 2, Index Auctorum, pp. 555-557; dove sono indicati anche i Padri della Chiesa e gli Scrittori ecclesiastici - centinaia di volte; mentre la Sacra Scrittura è citata circa 12.500 volte: vedi Praefatio, vol. 1, pp. IX e XIV. 
no-biblijnej i duchowej Doktora Anielskiego. Ta myśl jest nadal bardzo aktualna i pozwala na ciągłe trwanie w nauce, moralności i duchowości Kościoła Chrystusowego.

\section{The fruits of the Holy Spirit - the Commentary of saint Thomas Aquinas on the Gal 5,22ss}

\section{Summary}

In the presentation of the subject - preceded by an Introduction, where the Aquinas is presented as Bible's Commentator - the author examines the saint Thomas' interpretations of saint Paul's texts regarding the theme of this research, confronted with the contemporary exegesis, and presents three points: 1 - The vices (Gal 5,19-21); 2 - The fruits of the Holy Spirit (Gal 5,22-23a); 3 - Against such there is no Law (Gal 5,23b; whit the interpretation of Gal 5,24-25). All this permits to the author to conclude that the doctrine of the fruits of the Holy Spirit presented by Saint Thomas in this Commentary is very clear, rich and remains valid and topical and permits to remain in the faith, morality and spirituality of the Church.

\section{Parole chiave}

Spirito Santo, vizi, virtù, frutti, doni, Legge, vita cristiana

\section{Słowa kluczowe}

Duch Święty, wady, cnoty, owoce, dary, Prawo, życie chrześcijańskie

\section{Keywords}

Holy Spirit, vices, virtues, fruits, gifts, Law, Christian life

\section{Bibliografia}

Betz H.D., Galatians, Herm, Fortress, Philadelphia 1979.

Brena G.L., Interpretazione antropologica di san Tommaso, in AA.VV., Tommaso d'Aquino nel suo settimo centenario, vol. 7: L'uomo, Ed. Domenicane Italiane, Napoli 1978, pp. 83-100, qui p. 89.

Biffi I., Teologia, storia e contemplazione in Tommaso d'Aquino, Jaca Book, Milano 1995.

Burton E.W., The Epistle to the Galatians, ICC, T. e T. Clark, Edinburgh 1921.

Burton E.W., A Critical and Exegetical Commentary on the Epistle to the Galatians, Edinburgh 1921.

Cardoletti P., Presentazione, in B. Lonergan, Conoscenza e interiorità. Il Verbum nel pensiero di s. Tommaso, Dehoniane, Bologna 1984, p. 10.

Cazelles H., Bouhot J.P., Pentateuco, Paideia, Brescia 1968.

Chenu M.D., Introduzione, Tommaso d'Aquino, La conoscenza di Dio, Messaggero, Padova 1982 . 
Chenu M.D., San Tommaso d'Aquino e la teologia, Gribaudi, Torino 1989.

Congar Y.M.J., Zarys dziejów teologii, in AA.VV., Tajemnica Boga, Poznań-Warszawa-Lublin 1965, p. 190.

Courth F., Der Gott der dreifaltigen Liebe, ed. polacca: Bóg trójjedynej miłości, Pallottinum, Poznań 1997, p. 15.

Dąbrowski W., La dottrina sul peccato originale nei commenti di san Tommaso d'Aquino alle lettere di san Paolo Apostolo, in 'Angelicum' 83 (2006), pp. 557-629, qui pp. 568$-589$.

Dizionario di teologia biblica, X. Leon-Dufour (dir.), Marietti, Genova 2001.

Dockery D.S., Frutto dello Spirito, in G.F. Hawthorne, R.P. Martin, D.G. Reid (a cura di), Dizionario di Paolo e delle sue lettere, San Paolo, Cinisello Balsamo 2000, pp. 652$-656$.

Duajat J., Święty Tomasz z Akwinu a nowożytna fizyka, in AA.VV., Aktualność św. Tomasza, Pax, Warszawa 1975, p. 12.

Fung R.Y.K., The Epistle to the Galatians, NICNT, Eerdmans, Grand Rapids 1988.

Gillièron B., Lessico dei termini biblici, Elledici, Leumann 2000.

Gratsch E.J., Manuale introduttivo alla Summa Teologica di Tommaso d'Aquino, Piemme, Casale Monferrato 1988.

W. Kasper, Der Gott Jesu Christi, ed. italiana: Il Dio di Gesù Cristo, Queriniana, Brescia 2008, p. 22.

Kruse C.G., Virtù e vizi, in G.F. Hawthorne, R.P. Martin, D.G. Reid (a cura di), Dizionario di Paolo e delle sue lettere, San Paolo, Cinisello Balsamo 2000, pp. 1605-1607.

Leon-Dufour X., Dizionario del Nuovo Testamento, Queriniana, Brescia 1978.

Mondin B., Dizionario enciclopedico del pensiero di san Tommaso d'Aquino, Ed. Studio Domenicano, Bologna 1991.

Mondin B., La cristologia di san Tommaso d'Aquino. Origine, dottrine principali, attualità, Urbaniana University Press, Vatican City 1997.

Müller G.L., Dogmatica cattolica. Per uno studio e la prassi della teologia, San Paolo, Cinisello Balsamo 1999.

Obermayer H., Speidel K., Vogt K., Zieler G., Piccolo dizionario biblico, Paoline, Cinisello Balsamo 1991.

Pera C., Le fonti del pensiero di s. Tommaso d'Aquino nella Somma Teologica, Marietti, Torino 1979.

Pesch O.H., Tommaso d'Aquino. Limiti e grandezza della teologia medievale. Una introduzione, Queriniana, Brescia 1994.

Richè P., Chatillon J., J. Verger, Lo Studio della Bibbia nel Medioevo latino, Brescia 1989.

Rossano P. (a cura di), Lettere di san Paolo, San Paolo, Cinisello Balsamo 1998.

Serenthà L., Teologia dogmatica, in Dizionario Teologico Interdisciplinare, Marietti, Torino 1977, vol. 1, pp. 262-278, qui p. 269.

Storia della Teologia nel Medioevo, vol. 2: La grande fioritura, G. D’onofrio (dir.), Piemme, Casale Monferrato 1996.

Swieżawski S., Święty Tomasz na nowo odczytany, Kraków 1983. 
Taurisano I., La vita e l'epoca di san Tommaso d'Aquino, Studio Domenicano, Bologna 1991.

Thomae Aquinatis, Super Epistolas S. Pauli Lectura (a cura di p. R. Cai OP, editio VIII revisa), 2 voll., Marietti, Torino-Roma 1953.

Tommaso d'Aquino, Compendio di Teologia, introduzione, traduzione e note a cura di P. Agostino Selva OP, Studio Domenicano, Bologna 1995.

Tommaso d'Aquino, Opuscoli spirituali. Commenti al Credo, al Padre nostro, all'Ave Maria e ai Dieci Comandamenti, traduzione e note a cura di P. Lippini, Studio Domenicano, Bologna 1999.

Tommaso d'Aquino, Summa Theologiae, Edizioni Paoline, Cinisello Balsamo 1988.

Torrell J.P., Tommaso d'Aquino. L'uomo e il teologo, Piemme, Casale Monferrato 1994.

Vanni U. (versione - introduzione - note), Lettere ai Galati e ai Romani, in Nuovissima versione della Bibbia, vol. 40, Paoline, Roma 1979.

Verger J., L'esegesi dell'università, in AA.VV., Lo studio della Bibbia nel Medioevo latino, Paideia, Brescia 1989.

Währisch H., Tessarolo A., Virtù, in L. Coenen, E. Beyreuther, H. Bietenhard (a cura di), Dizionario dei concetti biblici del Nuovo Testamento, Dehoniane, Bologna 1991, pp. 1989-1993.

Walz A., De genuino titulo 'Summae theologiae', in 'Angelicum', 18 (1941), pp. 142-151. Weisheipl J.A., Tommaso d'Aquino. Vita, pensiero, opere, Jaca Book, Milano 1994.

Zedda S., Lettera agli Ebrei. Introduzione, in P. Rossano (a cura di), Lettere di san Paolo, San Paolo, Cinisello Balsamo 1998.

Zedda S., Prima Lettura di S. Paolo, Torino 1964. 